\title{
Comparing simulations and observations of the Ly alpha forest. I. Methodology
}

\section{Citation}

Petry, Cathy E., Chris D. Impey, Neal Katz, David H. Weinberg, and Lars E. Hernquist. 2002. "Comparing Simulations and Observations of the Lya Forest. I. Methodology." The Astrophysical Journal 566 (1): 30-50. https://doi.org/10.1086/338033.

\section{Permanent link}

http://nrs.harvard.edu/urn-3:HUL.InstRepos:41381700

\section{Terms of Use}

This article was downloaded from Harvard University's DASH repository, and is made available under the terms and conditions applicable to Other Posted Material, as set forth at http:// nrs.harvard.edu/urn-3:HUL.InstRepos:dash.current.terms-of-use\#LAA

\section{Share Your Story}

The Harvard community has made this article openly available.

Please share how this access benefits you. Submit a story.

Accessibility 
Submitted to the Astrophysical Journal

\title{
Comparing Simulations and Observations of the Lyman- $\alpha$ Forest \\ I. Methodology
}

\author{
Cathy E. Petry and Chris D. Impey \\ Steward Observatory, The University of Arizona, Tucson, AZ 85721 \\ cpetry@as.arizona.edu, cimpey@as.arizona.edu \\ Neal Katz \\ Department of Astronomy, University of Massachusetts, Amherst, MA 01003 \\ nsk@kaka. astro.umass . edu \\ David H. Weinberg \\ Department of Astronomy, The Ohio State University, Columbus, OH 43210 \\ dhw@astronomy . ohio-state.edu \\ and
}

Lars E. Hernquist

Department of Astronomy, Harvard University, Cambridge, MA 02138

lars@cfa.harvard.edu 


\begin{abstract}
We describe techniques for comparing spectra extracted from cosmological simulations and observational data, using the same methodology to link Lyman$\alpha$ properties derived from the simulations with properties derived from observational data. The eventual goal is to measure the coherence or clustering properties of Lyman- $\alpha$ absorbers using observations of quasar pairs and groups. We quantify the systematic underestimate in opacity that is inherent in the continuum fitting process of observed spectra over a range of resolution and $S N R$. We present an automated process for detecting and selecting absorption features over the range of resolution and $S N R$ of typical observational data on the Lyman- $\alpha$ "forest". Using these techniques, we detect coherence over transverse scales out to $500 h_{50}^{-1}$ $\mathrm{kpc}$ in spectra extracted from a cosmological simulation at $z=2$.
\end{abstract}

Subject headings: intergalactic medium - large-scale structure of the universe - methods: data analysis, N-body simulations - quasars: absorption lines

\title{
1. INTRODUCTION
}

The numerous lines of Lyman- $\alpha$ absorption that appear in the spectra of quasars are proving to be excellent cosmological probes. Hydrodynamic simulations of the universe reveal an evolving network of sheets, filaments and halos caused by complex gravitational dynamics in the expanding universe. The Lyman- $\alpha$ "forest" only identifies the neutral component of the intergalactic medium, but the absorbers are well understood tracers of the overall mass distribution (Hernquist et al. 1996; Miralda-Escudé et al. 1996; Rauch 1998). The simulations show that the physical state of the diffuse gas causing Lyman- $\alpha$ absorption is relatively simple, enabling the use of analytic models to relate the absorption to the underlying mass 
and velocity fields (McGill 1990; Bi 1993; Gnedin \& Hui 1996). The combination of theory and simulation has allowed the Lyman- $\alpha$ forest to be used for measurements of the baryon fraction (Rauch et al. 1997; Weinberg et al. 1997), the mass density (Weinberg et al. 1999), the amplitude of mass fluctuations (Gnedin 1998; Nusser \& Haehnelt 2000), the mass power spectrum (Croft et al. 1998, 1999, 2001; McDonald et al. 2000), the thermal history of the IGM (Haehnelt \& Steinmetz 1998; McDonald et al. 2000; Ricotti, Gnedin, \& Shull 2000; Schaye et al. 2000), the chemical evolution of the Universe (Cen \& Ostriker 1999; Aguirre et al. 2001,?), and the metallicity of the IGM (Rauch et al. 1997; Hellsten et al. 1997; Davé et al. 1998).

On the observational side, the detection of coincident absorption lines in the spectra of quasar pairs provided the first evidence that Lyman- $\alpha$ absorbers have a large transverse extent (Dinshaw et al. 1994; Bechtold et al. 1994; Dinshaw et al. 1998; Crotts \& Fang 1998). In principle, this idea can be extended with the use of quasar groups to thread a contiguous volume and provide 3D "tomography" of the absorbers, revealing the variation of coherence and homogeneity with redshift. In practice, however, quasars of the appropriate brightness, redshift, and angular separation are hard to find. Moreover, at $z \lesssim 2$, the opacity drops steadily and the Lyman- $\alpha$ forest thins out into a savannah, in agreement with numerical simulations (Davé et al. 1999). Over most of the Hubble time, the absorbers can only be observed in the vacuum ultraviolet with the twenty times smaller collecting area of the Hubble Space Telescope (HST). Studies of the low redshift Lyman- $\alpha$ forest are still very much limited by the quality of the available data.

Figure 1 summarizes the state of observational capabilities for studies of the Lyman- $\alpha$ forest. The boxes give the approximate bounds in resolution and $S N R$ of several different instrument and telescope combinations. Open boxes show examples of ground-based facilities that can only measure Lyman- $\alpha$, at $z \gtrsim 1.6$; shaded boxes show past, present and future 
HST instruments. The horizontal bar shows the range of observed Doppler parameters of the absorbing gas, which is independent of redshift (Penton, Shull, \& Stocke 2000). Only echelle observations are sensitive to the thermal and hydrodynamic properties of the absorbing gas. The best quality data come from Keck/HIRES and VLT/UVES. At lower redshift, the STIS echelle mode will be supplanted by the Cosmic Origins Spectrograph (COS). There is a large jump in resolution down to the level (1-2 $\AA$ ) where a larger number of targets is available and a larger redshift path length can be surveyed. The Keck/LRIS region is typical of most moderate resolution ground-based data. The volume of existing data will be eclipsed by the $\sim 10^{4}$ high redshift quasar spectra that will emerge from the Sloan Digital Sky Survey (York et al. 2000). At lower redshift, the largest single data set comes from the HST Quasar Absorption Line Key Project (Jannuzi et al. 1998; Weymann et al. 1998).

The goals of this paper are to (a) define a set of automated procedures that can be used to analyze data (with a wide range of spectral resolution, $S N R$, and physical separations between paired lines of sight) and spectra extracted from simulations, (b) form a bridge between the historically different methodologies of observers and simulators, and (c) facilitate the comparison between observations and simulations of the Lyman- $\alpha$ forest, in order to derive cosmological constraints.

The major challenge to the first goal is the fact that absorption features in a quasar spectrum must be measured with respect to an unknown continuum, and in the presence of broad emission features. This is usually accomplished with a low-order function fitted to the continuum, but no procedure is fully robust in dealing with broad absorption features or the region just blue-ward of the Lyman- $\alpha$ emission line. Even the comprehensive software of the HST Key Project cannot automatically deblend complex spectral regions (Schneider et al. 1993). By contrast, spectra extracted from the hydrodynamic simulations yield opacities directly with respect to a predetermined continuum. In addition, observers have traditionally 
treated absorption in terms of discrete features modeled by Voigt profiles, which in turn must be convolved with the instrumental response function. This approach is observationally successful, particularly at low redshift where spectral features have low number density and appear isolated in the spectra. Regardless of whether or not a Voigt profile provides a suitable description of the absorbing gas, there is evidence from extracted simulation spectra that much of the opacity is associated with shallow and smoothly varying absorption. This is commonly referred to as the fluctuating Gunn-Peterson approximation (Hernquist et al. 1996; Weinberg, Katz, \& Hernquist 1998).

With regard to the second goal, there are substantial differences between the extraction of information from optical or UV spectra, and the the extraction of information from a spectrum created as a product of a cosmological simulation. Observational spectra have finite $S N R$ and resolution, each one spans a large 1-dimensional redshift range, and the Lyman- $\alpha$ lines must be identified in spectral regions contaminated with metal lines from higher column density absorbers. Details of the method for culling out metal lines from the Lyman- $\alpha$ forest will be given in Paper II. Spectra extracted from hydrodynamic simulations have no noise and extremely high resolution, although the spectral features result from gas kinematics that map onto velocity space in a complex way. By contrast to the $\sim$ Gpc or larger 1D path of the Lyman- $\alpha$ forest in a quasar spectrum, the simulation boxes span 20-40 $h_{50}^{-1} \mathrm{Mpc}$ and are aliased for large scale structure measurements on scales larger than 5-10 $h_{50}^{-1} \mathrm{Mpc}$. However, the simulations are ideal for 3D measures of the gas distribution since $\sim 10^{5}$ independent spectra can be extracted, whereas 3D observations of absorber structure are limited by the rarity of suitable quasar pairs.

The third goal of this project will be realized with the second and subsequent papers in this series. In the second paper, we will compare coherence measures of absorbers at $z \sim 2$ with extractions from simulations. In future papers, we will extend the work to lower 
redshifts and investigate the dependence of the predicted absorber properties on cosmological parameters. This paper sets the stage by describing the techniques for automated and consistent comparisons between observations and simulations of the Lyman- $\alpha$ forest. In section 2, we discuss the detection and selection of absorption features. In section 3, we discuss the measurement of physical properties of the absorbers. In section 4, we discuss results from single and multiple sight-lines, including a comparison between line counting and measures that use the entire information contained in the spectra. Section 5 contains a brief summary.

\section{ABSORPTION LINE SELECTION}

\subsection{Extracting Spectra from the Simulation}

A numerical simulation of a $z=2$ cold dark matter (CDM) universe, with $H_{0}=50 \mathrm{~km}$ $\mathrm{s}^{-1} \mathrm{Mpc}^{-1}$, normalized to yield a present-day rms mass fluctuation of $\sigma_{16}=0.7$ in spheres of radius $16 h_{50}^{-1} \mathrm{Mpc}$, was performed by Hernquist et al. (1996) using a method based on smoothed-particle hydrodynamics, or SPH (Hernquist \& Katz 1989; Katz, Weinberg \& Hernquist 1996). The simulation volume is a periodic cube of comoving size $22.2 h_{50}^{-1} \mathrm{Mpc}$ drawn randomly from a CDM universe with $\Omega_{m}=1$ and baryon density $\Omega_{b}=0.05$. The simulation includes the effects of a uniform photoionizing radiation field, where radiative heating and cooling rates are computed assuming optically thin gas in ionization equilibrium with this field. There are $64^{3} \mathrm{SPH}$ particles and an equal number of dark matter particles; the masses of the individual particles are $1.45 \times 10^{8} M_{\odot}$ and $2.8 \times 10^{9} M_{\odot}$, respectively. The gas resolution varies from $\sim 5 \mathrm{kpc}$ in the highest density regions to $\sim 200 \mathrm{kpc}$ in the lowest density regions. Although more recent simulations have been performed with higher spatial resolution and with the parameters of the currently favored cosmological model $\left(H_{0}=70\right.$ $\left.\mathrm{km} \mathrm{s}^{-1} \mathrm{Mpc}^{-1}, \Omega_{m}=0.35, \Lambda=0.65\right)$, most of the issues that relate to comparisons with 
observations can be illustrated with this single simulation.

Spectra are extracted from the simulation cube by computing the imprint of the density, temperature, and velocity of the neutral gas fraction associated with each SPH gas particle

on a flat continuum (for more details, see Katz et al. 1996). One thousand sets of spectra were extracted from the simulation cube by randomly selecting 1000 positions from the $\mathrm{x}$ projection. For each of the 1000 primary lines of sight (PLOS), six adjacent spectra were extracted that are offset $33.3,100,233,400,667$, and $1000 h_{50}^{-1} \mathrm{kpc}$ in proper distance from each PLOS. The azimuth angle of the adjacent spectra was varied randomly from one primary sightline to another. This strategy allows the examination of coherence of the absorption features in the extracted spectra over these (and other intermediate) transverse separations, in addition to enabling comparisons with observational data for quasar pairs having a range of separations. The transverse separations between the lines of sight range from less than the resolution of the simulation to a level where absorber coherence is expected to be very weak.

Each spectrum is sampled with 1000 data points spanning $1924.5 \mathrm{~km} \mathrm{~s}^{-1}$, which corresponds to a spectral range of $23.4 \AA$ at $z=2$. Figure 2 shows six spectra extracted from the simulation illustrating the range of absorption features, with the mean opacity increasing going from panel $(a)$ to $(f)$. Panels $(c)$ and $(d)$ have a typical opacity for extracted spectra at this redshift, and less than $1 \%$ of the sight-lines are as heavily absorbed as the example in panel $(f)$.

\subsection{Degrading the Extracted Spectra}

One objective of this paper is to compare absorber properties measured from the observed spectra to those measured from the spectra extracted from the simulation. For a fair 
comparison to be made, the extracted spectra are processed to reflect the inevitable limitations of observational data due to instrumental effects of noise and resolution. The spectra are extracted from the simulation cube in terms of optical depth as a function of velocity, and are converted to transmission, $T=e^{-\tau}$, as a function of wavelength in Angstroms, $\lambda_{i}=\lambda_{L y \alpha}(1+z)\left(1+v_{i} / c\right)$, where $z=2$.

As can be seen in Figure 1, the bulk of the UV data on the Lyman- $\alpha$ forest, which contains information about the IGM over the last three-quarters of the age of the universe, is of rather modest $S N R$ and resolution. This means that the complex dynamical processes that imprint themselves on the absorber profile are not visible at low redshift, even in the few published echelle spectra (Penton, Stocke, \& Shull 2000; Tripp, Savage, \& Jenkins 2000). With non-echelle spectra, the spectral profile is dominated by the (typically) Gaussian shape of the instrumental profile, and Doppler parameters cannot be measured. For the purposes of this paper and the following Paper II, we chose a range of values for the resolution, $\Gamma_{\text {res }}$, and $S N R$ typical of most published quasar spectra. To span the parameter space of available data at any redshift we chose $S N R=10,30$, and 100, and $\Gamma_{r e s}=5,20,80$, and $300 \mathrm{~km} \mathrm{~s}^{-1}$, and form twelve realizations of a single spectrum extracted from the simulation, each with a different combination of $S N R$ and resolution.

The extracted spectra are convolved with a Gaussian profile having a FWHM, $\Gamma_{r e s}$, with each of the four values chosen to represent the instrumental resolution of the available observational data. The velocity resolution of the extracted spectra $\left(\sim 2 \mathrm{~km} \mathrm{~s}^{-1}\right)$ is much higher than the resolution of observed quasar spectra, the range of which is represented by the four values $5,20,80 \& 300 \mathrm{~km} \mathrm{~s}^{-1}$. We resample the extracted spectra to 3 per resolution element by spline interpolation. Poisson noise is added to each spectrum by scaling the intensity at each pixel to the $(S N R)^{2}$, adopting a Poisson deviate with that value as the mean, and then renormalizing the spectrum. 
Figure 3 shows two different extracted spectra each having an opacity similar to the mean at $z=2$, exemplifying the typical absorption morphologies at that redshift. The twelve realizations for each individual extracted spectrum show the degraded appearance due to the various choices of $S N R$ and resolution. The highest resolution, highest $S N R$ spectrum (top right panel in each case) is a fairly close match to the original spectrum

extracted from the simulation. The best published spectra from the Keck telescopes appear like those in the top right panels, but there are a significant number of HST spectra that are similar in quality to the lower left panels. These plots clearly show the separate effects of $S N R$ and resolution on the appearance of the spectrum.

\subsection{Fitting Continua to the Extracted Spectra}

The measurement of absorption features in quasar spectra, whether by line profile fitting or by using a type of flux statistic, must be performed relative to the quasar continuum. Continuum fitting is generally done by iteratively fitting the data points in a spectrum, eliminating negative or downward excursions from the fit, and refitting. The position of the final adopted continuum depends on the $S N R$ of the spectrum and, more importantly, on the total flux absorption (which is directly related to the line density) and its distribution across the spectrum. Such a procedure only approaches the "true" continuum in the limit of infinite signal to noise, and even then has the potential to miss weak or shallow absorption features. Figure 3 demonstrates how features that can be seen clearly in the highest $S N R$, highest resolution spectra are lost in lower $S N R$, lower resolution spectra.

Given that there are no regions of truly zero absorption, it is essentially guaranteed that line and continuum fitting will miss some portion of the Lyman- $\alpha$ opacity. The systematic underestimation of the continuum level will also tend to underestimate the equivalent width and therefore the column density of fitted lines too. We use extracted spectra from the 
simulation to calibrate the size of this effect, and measure it as a function of $S N R$ and resolution.

With many thousands of spectra to process, an automated procedure is required. After the simulation spectra are manipulated to mimic observational data, the continuum must be estimated as for the observed spectra. Any algorithm must be simple conceptually and should be able to fit continua automatically for spectra with a range in absorber opacity corresponding to a redshift range of $0<z<3$, and for spectra having a range of signal-tonoise ratios, $10<S N R<100$. Any method implicitly assumes that the data retains some of the quasar's emission continuum, and this implies that continua fit to highly absorbed regions will be less representative of the true continuum. Mean absorption opacity and $S N R$ are the primary factors in determining how well the true (or input) continuum can be recovered.

Fitting a cubic spline is a standard method of estimating continua for observed spectra (Schneider et al. 1993). Splines offer the advantage of being able to fit a smooth, continuous function through a large number of data points. In quasar spectra, the large available wavelength range acts to define a smooth, slowly-varying continuum. However, the simulation volume offers only a short spectral range, and spline fitting to short extracted spectral segments with significant absorption can produce steeply-sloped continua. We suppress this effect in the case of the extracted spectra by "tripling" the spectrum, or lining up three identical spectra, taking advantage of the fact that the simulation cube is periodic. This is merely a practical mechanism for dealing with the edge effects in continuum and absorption line fitting - for the analysis, only lines whose centers fall in the central third of the new spectrum (the original extracted spectrum) are included.

To estimate a continuum computationally, representing what one might fit "by eye," we implement a two step process. The first step is to iteratively fit a straight line to $23 \AA$ sections 
of a spectrum (corresponding to the independent spectral segment length for the extracted spectra), providing an estimate of the amplitude of the continuum over that wavelength range. This is a reasonable way to proceed because emission continua of quasars can be closely approximated by series of linear segments over $\sim 20 \AA$ scales, except in the vicinity of Lyman- $\alpha$ emission. All data points are weighted equally and an iterative process rejects points that deviate negatively by $2 \sigma$ from the current fit, excluding them from subsequent fits. The rejection process converges after only four cycles.

The method of continuum fitting used by the HST Absorption Line Key Project divides a spectrum into bins and fits a spline to the average flux in these bins. However, this bin size is larger than the length of our extracted simulation spectra, so the first step described above provides our first estimate to the continuum. The second step produces a smoothly varying continuum over the length of the spectrum using the amplitude of each of the fitted line segments from the first step. A single point is created for each of the three segments by evaluating the amplitude of the fitted straight line at the average wavelength of each segment. A cubic spline is fit through these points for the length of the spectrum. Visual inspection of spectra with a range of flux decrements demonstrates that this two step method results in a more satisfactory fit to the continuum than the Key Project method, especially for heavily absorbed spectra, as the first step has the effect of "floating" the continuum to what appears "by eye" to be a more appropriate level.

To evaluate how well the algorithm works for the observational spectra, we compare the final fitted continua using the new two-step process to the continuum fit using the method used by the HST Absorption Line Key Project. Some manual adjustment of the continuum is necessary in the vicinity of the steeply sloped Lyman- $\alpha$ emission features. Four of the six spectra to be analyzed in Paper II of this series - PG 1343+264A,B, LB 9605, and LB 9612 — were studied using both methods, and the ratio of the two fitted continua is unity at 
all wavelengths to well within the errors. This demonstrates that no significant systematic effects are introduced by the continuum fitting algorithm. We can also infer that absorption line parameters returned by the two methods will not significantly differ.

To show how $S N R$ and resolution affect the level of the fitted continuum, we fit continua using our method to 1000 randomly-selected spectra extracted from the simulation. We then compare the mean opacity computed for each of 1000 undegraded (i.e. raw) spectra extracted from the simulation to the mean opacity calculated relative to the fitted continuum for each degraded spectra in each of the 12 realizations of $S N R$ and $\Gamma_{\text {res }}$. Figure 5 shows the distribution of the difference of these two quantities for all 1000 spectra and for each realization. This difference is equivalent to the difference between the input continuum level (unity, by definition) and the fitted continuum level, converted into an opacity decrement, $\Delta \tau$.

An opacity decrement is the unavoidable consequence of any fitting procedure where the continuum is not known a priori - as is the case for all observational data. The quantity $\Delta \tau$ therefore represents "lost" opacity, and it has two components. The first is due to the fact that strong absorbers will have their column densities slightly underestimated owing to the lower placement of the continuum. The second is due to the sum of broad and/or low level absorption that cannot be recovered by fitting a noisy continuum (the Gunn-Peterson approximation). See Figure $2 d$ for a good example.

Figure 4 shows how the severity of underestimating opacity depends on both $S N R$ and resolution by comparing the mean opacity measured for the undegraded spectra to the mean opacity measured relative to the fitted continuum for the degraded spectra. In Figure 5, we plot the distribution of the difference of these two quantities, $\Delta \tau$. At the level of the highest quality echelle data (top-right panel), 80-90\% of the absorption opacity is recovered in almost every case. At the level of poor quality HST data (lower-left panel), the opacity 
underestimation is typically $50 \%$. The tail to high $\Delta \tau$ in each panel represents the relatively rare cases of heavily absorbed spectra, where no automated procedure can adequately recover the absorption.

\section{ABSORPTION LINE MEASUREMENT}

\subsection{Line Measurement and Deblending}

We have examined the general effects of a wide range of resolution and $S N R$ on the appearance of the spectra and on the ability to recover the true continuum level. For the phase of absorption line measurement, we home in on a narrower range of parameter space with the four combinations: $S N R=10$ and 30 , and resolution $\Gamma_{r e s}=80$ and $300 \mathrm{~km} \mathrm{~s}^{-1}$. It can be seen from Figure 1 that these values bracket most existing HST data and much of the anticipated data from the Sloan quasar survey. This range also encompasses the data we will use in Paper II for a Lyman- $\alpha$ coherence measurement at $z=2$. Despite the conceptual limitations of the line/continuum fitting paradigm, we acknowledge the fact that a huge amount of observational data has been published using this type of procedure. A major goal of this paper is to form a bridge between the appropriate techniques used to analyze simulations and the traditional methods of quasar spectroscopy.

\subsubsection{The Absorption Line Profile}

Available quasar pair data on the Lyman- $\alpha$ "forest" have $S N R$ and resolution generally no better than $S N R=30$ and $\Gamma_{r e s}=80 \mathrm{~km} \mathrm{~s}^{-1}$. This corresponds to the highest $S N R$ and resolution of the four parameter spaces for which we are measuring absorption lines in the extracted spectra. Dectection of the weakest lines is limited by the $S N R$ and the resolution of the data, and measurement of the column density depends on the Doppler parameter. 
However, integration of the evolution function for Lyman- $\alpha$ absorbers shows that less than $1 \%$ of absorbers are expected to have column densities higher than $\log N=15$ (Scott, Bechtold, \& Dobrzycki 2000). The range of Doppler parameters for Lyman- $\alpha$ absorbers at $z \sim 2$ spans approximately 20-80 $\mathrm{km} \mathrm{s}^{-1}$ (Hu et al. 1995). For an absorber with a median Doppler value $b=30 \mathrm{~km} \mathrm{~s}^{-1}, \log N=15$, and $\Gamma_{r e s}=80 \mathrm{~km} \mathrm{~s}^{-1}$, the line profile is just saturated or reaches zero flux at the line center. For higher resolution data, line-fitting methodologies represent individual absorbers with a Voigt profile, but we demonstrate that for column densities less than $\log N=15$ and resolutions lower than $\Gamma_{r e s}=80 \mathrm{~km} \mathrm{~s}^{-1}$, the use of a Gaussian profile is justified.

We generate the flux profile for a Lyman- $\alpha$ absorption line (with essentially infinite $S N R$ and resolution) using subroutines from the program AutoVP (Davé et al. 1997) for the case of $\log N=15$ and $b=80 \mathrm{~km} \mathrm{~s}^{-1}$. The convolution of this "intrinsic" line profile with the instrumental line spread function (a Gaussian with $\Gamma_{r e s}$ ) is the expected flux line profile. Sampling of the dispersion is chosen to mimic that of the degraded simulation spectra for this parameter space.

One thousand absorption lines were created and Poisson noise was added to represent $S N R=30$. A Gaussian profile was fitted to each Monte Carlo simulated line. This fitted Gaussian profile was compared to the Monte Carlo line profile, yielding an average $\chi^{2}$ value of 0.011. For 50 degrees of freedom (the number of data points in the profile), this corresponds to a probability of less than $10^{-5}$ that the two profiles are different. We also note that low resolution $\left(\Gamma_{\text {res }}>80 \mathrm{~km} \mathrm{~s}^{-1}\right)$ renders undetectable all the complexities in the absorber profile caused by hydrodynamic effects. The use of a Gaussian profile in fitting absorption features is therefore adequate for our immediate purposes, and for our initial science application in Paper II. 


\subsubsection{Line Selection Algorithm}

Absorption line selection and measurement is performed using software originally written by Tom Aldcroft (Aldcroft 1993) that has since been substantially revised. The most recent version of the software retains the original code structure, the graphic interface tools, and the fundamental numerical subroutines. New methodology for fitting continua, and new algorithms for selecting and fitting absorption features have been implemented. The continuum-fitting process is described in the previous section. Line selection and fitting is a two-step process where a preliminary list of lines is selected, which forms a first estimate to the simultaneous fit that is determined in a subsequent step. The line selection and fitting methodology was first described in Petry, Impey, \& Foltz (1998). Some refinements to the line fitting algorithm have been added to generalize finding the techniques for a range of data quality and to eliminate manual intervention. The range of data quality addressed in this work is $S N R=10$ to 30 and $\Gamma_{\text {res }}=80$ to $300 \mathrm{~km} \mathrm{~s}^{-1}$.

Selecting a combination of absorption lines profiles to fit an absorption region is a difficult because of the effects of instrumental resolution and $S N R$ on the true features in the spectrum. The second extraction in Figure 3 shows an example (at $\sim 3662 \AA$ ) where lines that are easily resolved at $20 \mathrm{~km} \mathrm{~s}^{-1}$ cannot be separated at a resolution $80 \mathrm{~km} \mathrm{~s}^{-1}$. The $S N R$ is not as important a factor in concealing the structure of an absorption feature until it becomes very low. No procedure can recover information that is lost due to limited instrumental resolution, but Gaussian profile fitting does provide a fair comparison between the simulation spectra and observed spectra. Even if a Gaussian is a good approximation to the shape of strong absorption features, we do not expect that line-fitting can yield unique fits. The biggest danger is expected to be over-fitting, such as when a single broad feature is fit by several overlapping Gaussians. However, it is also possible to under-fit, such as when noise causes a weak feature to fall below the significance threshold for a single unresolved 
line. In what follows, we describe a robust procedure for dealing with most of the situations presented by real quasar spectra.

One simple way of fitting a number of Gaussian profiles to a predefined absorption region is to fit the peak of a Gaussian to the lowest flux point in the spectrum, subtract the profile from the spectrum, and then repeat the process at each local minimum. The method is considered to have converged when the result is consistent with the flux error of the original spectrum. However, with this method, a region which may be better fit in a $\chi^{2}$ sense by two barely resolved lines, will actually be fit by one strong line in the center, possibly with an unphysically large width, straddled by two smaller "satellite" lines. The sequential approach has the basic problem that lines are defined one at a time, which constrains the choice of subsequent lines, and does not ensure an optimal or unique fit.

We have devised an iterative method to fit the maximum number of lines to a region and distribute the lines optimally across the region. Unresolved lines are assumed, i.e. the absorber is well approximated by the instrument line profile (a Gaussian). Subsequently, all combinations of lines from this preliminary list are fit simultaneously to the region using a Marquardt minimization technique, and the fit that meets a preset criteria is chosen as the best fit. We consider that the best fit is the deepest depression in the $\chi^{2}$ surface of solutions. The Marquardt minimization does not allow for large changes in the line center or line width; by design, the preliminary step of finding lines and subsequent testing each combination of lines samples the surface of solutions well enough to find the best solution. This is justified in practice because the iterative procedure rarely leads to substantial changes in the centers and widths of the strongest features.

In the first phase of line selection, an iterative search is made for all minima in the data array containing the flux values for each spectrum that has been convolved with the instrumental profile. A primary line list is formed from minima found in the first pass and a 
secondary list is made from minima found in the convolved array after lines in the primary list are subtracted from the original data. The primary and secondary lists combine to form the preliminary line list that defines an initial "best guess" at the final line list. The rationale for combining primary and secondary lists is as follows. The minima in the data array locate the strongest absorption features, but these inflection points do not account for all the absorption in a blended region. The secondary list serves to "interleave" the primary list, and combination of the two yields a fairly complete and robust map of the absorbers.

The iterative method for initially locating and measuring lines in the spectrum of each component follows that described in detail by Paper II of the HST Quasar Absorption Line Key Project (Schneider et al. 1993). The equivalent width, $W$, at every pixel in each spectrum is computed by centering the spectral line profile on the $i^{\text {th }}$ pixel and performing the weighted sum over the $6 \sigma$ limits of the Gaussian forming the array $W_{i}$. Similarly, this is done for the error array, $\sigma W_{i}$, and the interpolated error array, $\bar{\sigma} W_{i}$. The interpolated error array is calculated by replacing flux errors for data points that deviate negatively by more than $2 \sigma$ by the average of the errors in up to five (on each side) of the adjacent continuum points. Continuum in this sense is defined by points lying within a $2 \sigma$ deviation from the fitted continuum. This array differs from the error array in that the errors at the centers of the absorption lines are increased slightly to reflect the noise in the adjacent true continuum. The $S N R$ of an unresolved line is conventionally defined as $W_{i} / \sigma W_{i}$, whereas we choose to define "significance" as $W_{i} / \bar{\sigma} W_{i}$, which is used to select preliminary lines in the first phase. This correction for the drop in the errors at the centers of absorption lines, where the flux errors are smaller, allows for a more uniform comparison of relative line strengths.

The primary line list is generated by identifying the minima in the $W_{i}$ array within a window of width one half the instrumental resolution that has a significance $\left(W_{i} / \bar{\sigma} W_{i}\right)$ of three or more. A parabolic fit through the two adjacent points identifies the line center and 
the value of $W_{i}$ and $\sigma W_{i}$ corresponding to this wavelength give the equivalent width and error. The profiles of the lines are calculated and subtracted from the original spectra. This subtracted spectrum is then convolved and a secondary line list was derived in the same way. The secondary list is then subtracted from the original spectrum and a pass was made again that refits only the primary lines and may add new lines or drop existing lines. The number of lines added by the secondary list increases by a factor of 1.5 to 2 times the number of lines found in the primary list.

It was found empirically that after approximately five iterations of finding lines for first the primary and then the secondary lists, the number of lines converged to a repeating series as the line centers shifted about slightly depending on the noise. The number of lines that are added or lost by all subsequent iterations is no more than a few percent of the total list. The preliminary line list was formed from the combination of the two lists, and if their profiles are subtracted from the original data array, the result has a mean flux of zero and variations entirely consistent with noise.

\subsubsection{Line Fitting Algorithm}

The primary list of lines is input as a first guess to the simultaneous best fit of each absorbed region in a spectrum. For practical reasons the spectrum is broken up into regions. There are several ways of doing this and we chose to step through each spectrum and define the region to be fit as beginning where the flux array downward-crosses the continuum and ending where it upward-crosses the continuum. This method of defining the spectral regions is reasonable for data of resolution and $S N R$ under consideration here, because the number

of upward- and downward-crossings divides the spectrum into sections that can be fit by a moderate number of preliminary lines, and because it limits the number of regions where no preliminary lines are found. Fitting all combinations of the preliminary lines makes it 
computationally impractical to fit more than 11 lines per region, so in the rare occasions that this situation occurs the region is split at the highest flux data point in the region, and the fit is performed for the new region with a smaller number of lines.

Lines from the initial list that fall within the region to be fit were counted, $n$, and the number of all possible combinations of these lines, $N_{c o m b}$, was computed by summing the binomial coefficient, $C\left(\begin{array}{l}n \\ x\end{array}\right)$, which gives the number of combinations possible for $n$ lines taken $x$ positions at a time, over the number of possible positions, $x=1 \ldots n$

$$
N_{\text {comb }}=\sum_{x=1}^{n} C\left(\begin{array}{l}
n \\
x
\end{array}\right)=\sum_{x=1}^{n} \frac{n !}{x !(n-x) !} .
$$

The derived lines for each individual combination were worked out and saved, and each combination of lines was fit simultaneously to the region following a Marquardt minimization technique that varies the amplitude, center and width of the lines. The line parameters and the reduced chi square $\left(\chi_{\nu}^{2}\right)$, which is later used to select the best fit, for each combination were saved. The method of fitting all combinations of preliminary lines optimizes selection of the best fit to any absorption feature by maximally utilizing the information provided by the preliminary line list.

However, in practice, $\chi_{\nu}^{2}$ is not a sensitive test of overfitting in selecting the best fit to a region (Rauch et al. 1992). For example, it is possible for a fit to produce a $\chi_{\nu}^{2}$ that is improved from the prior fit but where the errors in the equivalent width are larger than the equivalent width itself. In our application of this procedure, the central wavelengths are fairly well determined and do not change more than a few standard deviations with subsequent fits. We therefore imposed secondary constraints to ensure that the final fit consisted of meaningful line parameters. The final "best" fit is chosen as the fit with the lowest $\chi_{\nu}^{2}$ that also fulfills the following criteria: (a) $\chi_{\nu}^{2} \leq 100$, (b) the errors in the fitted equivalent width and the FWHM are smaller than the measurement itself, (c) the minimum line separation for lines in a fit is $\Delta \lambda=1.12 \AA$, and (d) $0.65 \AA<\mathrm{FWHM}<4.5 \AA$. 
The first two of these constraints apply generally to the fitting procedure. The restriction on $\chi_{\nu}^{2}$ is an empirically determined practical limitation to the largest value expected to be associated with a reasonable fit. The best fit can have a $\chi_{\nu}^{2}$ that is rather high, simply due to the fact that there may be a portion of the fit region where no lines are fit but where the normalized flux is not exactly at the continuum level. Failure of this criterion forces a redetermination of the fitted region by dividing it at the wavelength having the next highest intensity, and a refitting of the preliminary lines to obtain a fit having a lower $\chi_{\nu}^{2}$. The restriction on the errors of fitted parameters to lines prevents over-fitting.

The last two constraints depend on the range of $S N R$ and resolution of the data being considered. In this case, the choices were justified as follows. The minimum and maximum FWHM values are determined by convolving the range of expected Doppler widths (Hu et al. 1995) with the instrumental profile, and allowing for variation in the minimum FWHM due to noise. The minimum line separation also depends on resolution and noise, and was chosen empirically by forming the distribution of line separations and then examining the reliability of individual fits. Instrumental resolution in this test case corresponds to 80 $\mathrm{km} \mathrm{s}^{-1}$. There is a natural break in the distribution at $\sim 70 \mathrm{~km} \mathrm{~s}^{-1}$ and lines selected with smaller separations than this represent an over-fit with respect the features in the undegraded simulation spectrum. Lines with separations of more than $\sim 90 \mathrm{~km} \mathrm{~s}^{-1}$ are reliably selected by the algorithm. For lines with separations between 70 and $90 \mathrm{~km} \mathrm{~s}^{-1}$ it is not clear by eye whether adding the second line to the fit is justified, and in all cases the undegraded simulation spectra shows an asymmetry or two features close together. A Monte Carlo simulation to determine the recovery rate of two close lines shows that the software does not reliably recover the input lines until they are separated by $\sim 100 \mathrm{~km} \mathrm{~s}^{-1}$. This motivates our minimum separation of $1.12 \AA$. However, we note that the statistical results of the analysis are not particularly sensitive to this exact choice. 
If the procedure fails one or more of the tests in any particular spectral region, the region is split at the next highest minimum intensity point and a new set of simultaneous fits is made using the smaller number of lines. If all of these fits fail, the last fit is chosen but is tagged. The tagged lines were examined for a subset of 200 spectra and were only $3 \%$ of the total number of lines selected. Two thirds of these were moderately broad, weak features whose shape had been distorted with the addition of noise - in general, a line was found in the vicinity of the true feature even if the addition of noise and resolution made it more difficult to detect. Almost all of the lines from "failed" fits are weak enough that they will not meet the selection criteria to be included in the subsequent analysis. About one in 5 were strong broad features where the chosen fit appeared appropriate, but the $\chi_{\nu}^{2}$ was too high. A remaining tiny number, barely $0.01 \%$ of the whole sample, are weak features fit to the edge of broader absorption regions or are not real features at all. This same visual inspection of many of the fits showed that under-fitting or over-fitting is not a significant problem for the algorithms. We conclude that the fraction of strong regions of absorption that are not well fit by the line/continuum algorithm is negligible.

The procedures for line-fitting are robust and can be applied in an automated way, but they are also somewhat complex. Figure 6 shows how they work in practice, as applied to the two extracted spectra from Figure 3, and in each of the four combinations of $S N R=10$ and $30, \Gamma_{r e s}=80$ and $300 \mathrm{~km} \mathrm{~s}^{-1}$. In other words, Figure 6 shows actual line/continuum fits to the four parameter choices at the lower left of Figure 3. Fitted continua and lines are overplotted in each case with the location of significant $\left(5 \sigma_{\text {det }}\right)$ absorbers shown by tick marks. "Significance" is defined in two ways: $\sigma_{\text {det }}$ is related to the flux error and is used to describe the strength of a line in terms of the detection limit of the data; $\sigma_{f i t}$ is the error in the equivalent width returned by the software and is a measure of line reliability or goodness of fit. In practice, $\sigma_{\text {det }}$ can be used to set a uniform detection threshold for absorbers and is directly related to the $S N R$, where $\sigma_{f i t}$ is an indicator of how well the equivalent width 
is determined - lines with large $\sigma_{\text {fit }}$ might be found in noisy regions of the spectrum or in the wings of broader absorption features. All lines fit by the software are retained regardless of strength or reliability, but for the analysis we define two categories: secure and marginal. The criterion for inclusion in either list is $5 \sigma_{\text {det }}$. Lines that are $<5 \sigma_{\text {fit }}$ make up the marginal list, and lines that have an equivalent width $\geq 5 \sigma_{f i t}$ constitute the secure list. Marginal lines may be reliable enough to be useful in the comparison with observed spectra, but in this work we only consider lines from the secure list in the analysis. In Figure 6, longer, thicker tick marks show the location of the secure lines, and shorter, narrower tick marks denote lines from the marginal category.

\section{PROPERTIES OF THE ABSORBERS}

\subsection{Line Counts Along Single Sight-lines}

Absorption features were measured using the line-fitting algorithm for 300 primary lines of sight (PLOS) and for their adjacent sight-lines spanning separations from 33.3 to 1000 $h_{50}^{-1} \mathrm{kpc}$ - a total of 2100 spectra extracted from the simulation. This was done for two different signal-to-noise ratios, $S N R=10$ and 30 , and two resolutions $\Gamma_{\text {res }}=80$ and 300 $\mathrm{km} \mathrm{s}^{-1}$, forming four realizations of the data. We compared the distribution of absorber properties measured from the simulated spectra to those found from observations of large samples of spectra published in the literature as a basic cross-check of our method.

In Figure 7, we show the distribution of the line centers of absorbers in 300 PLOS of the $S N R=30, \Gamma_{r e s}=80 \mathrm{~km} \mathrm{~s}^{-1}$ realization for each of the three projection axes. The deviations from the mean (shown as the dotted line) indicate large scale, coherent structures within the simulation box, having filamentary and sheetlike properties. However, the average of the 3 projections converges toward the mean value indicating that the absorbers approach a 
uniform distribution over the full size of simulation box, and demonstrating that there are no artifacts introduced by edge effects. Additionally, we examine the distribution of the number of absorbers per line of sight in Figure 8. This distribution is consistent with a Gaussian distribution that is the expectation for a random distribution of absorbers. In particular, the high multiplicity of absorbers that would indicate substantial clustering is not seen.

Table 1 lists the average number density $(d N / d z)$ of secure lines per line of sight above a limiting rest equivalent width threshold $\left(5 \sigma_{d e t}\right)$ for each of the four realizations of the simulated spectra (column 5). For Sample 1 (column 1) we used 1000 PLOS for increased statistics on the highest $S N R$ and resolution realization, and for the other three samples we used 300 PLOS. The number of lines from observations is obtained in two ways from a study performed by Scott, Bechtold, \& Dobrzycki (2000). These values are listed in columns 6 and 7 of Table 1. First, we simply count the number of absorbers found in the small wavelength range spanned by the simulated spectra at $z=2$ (J. Scott, private communication). However, a limited number of absorbers are found in this $23 \AA$ wide region and so the error bars are large. Since the evolution function (described below) is close to linear over $2<z<2.1$, the Poisson error can be reduced by using the lines counted in this five times larger spectral region as an estimate for the smaller spectral region actually spanned by the simulation.

Second, the average number of lines at $z=2$ can be computed from coefficients of an evolution function fitted to an appropriate sample of observed absorbers. This method is less direct but it has the merit of incorporating most of the available data. The number of Lyman- $\alpha$ lines per unit redshift per unit equivalent width can be described as follows:

$$
\frac{\delta^{2} \mathcal{N}}{\delta z \delta W}=\frac{A_{0}}{W^{*}}(1+z)^{\gamma} \exp \left(-\frac{W}{W^{*}}\right) \text {. }
$$

Integrating Equation 2 with respect to $W$ gives the number of lines per line of sight with a rest equivalent width greater than $0.16 \AA$ for the chosen values $\mathcal{A}_{0}=5.86, W^{*}=0.257$, and 
$\gamma=2.42$ from Scott, Bechtold, \& Dobrzycki (2000):

$$
\frac{\delta \mathcal{N}}{\delta z}=\mathcal{A}_{0}(1+z)^{\gamma}, \text { where } \mathcal{A}_{0}=\mathrm{A}_{0} \exp \left(-\frac{\mathrm{W}}{\mathrm{W}^{*}}\right)
$$

Further integration with respect to $z$ gives the number of lines per spectrum in the range $z_{\min }<z<z_{\max }$ :

$$
N=\frac{\mathcal{A}_{0}}{\gamma+1}\left[\left(1+z_{\max }\right)^{(\gamma+1)}-\left(1+z_{\min }\right)^{(\gamma+1)}\right] .
$$

The number of lines in this redshift interval for a different equivalent width limit, $w_{\text {lim }}$, is obtained by scaling $N$ by the factor $\exp \left[-\frac{\left(w_{l i m}-0.16\right)}{W^{*}}\right]$. Errors on $N$ are obtained using the $1 \sigma$ error bars on $\gamma$. See column 7 in Table 1 .

A comparison is made only for the $\Gamma_{\text {res }}=80 \mathrm{~km} \mathrm{~s}^{-1}$ realizations (Samples 1 and 3), because this resolution closely matches the data set used by Scott, Bechtold, \& Dobrzycki (2000). The error bars listed in Table 1 are merely reflect the Poisson error and do not include systematic uncertainties due to differences in the line-fitting algorithms that can be as large as 10-20\%. Sample 3 most closely matches the sensitivity of the data set used by Scott, Bechtold, \& Dobrzycki (2000), and the number of lines found in the extracted spectra agree to within $20 \%$ with both methods for counting observed lines. Sample 1 in Table 1 agrees with the (poorly-determined) number predicted using a model for the evolution of the absorbers, and it also agrees to within $20 \%$ of the expected number from the direct line counting method.

In addition to a check of aggregate line counts, it is important to check whether extracted spectra from the simulation return the observed distribution of line strength. We therefore compare the number of lines per equivalent width bin, $d N / d W$, to a measurement that uses the data of Scott, Bechtold, \& Dobrzycki (2000). This recent study provides better statistics at $z=2$ than the Hubble Space Telescope Absorption Line Key Project (Bahcall et al. 1993; Weymann et al. 1998). Figures 9 and 10 are plots of the number of secure $\left(\geq 5 \sigma_{f i t}\right)$ absorbers 
counted in the spectra from the simulations, binned by rest equivalent width, for Samples 1

and 3. Both distributions are well within the $1 \sigma$ error bars on $\gamma$, showing that the absorber statistics obtained by line-fitting applied to the simulation spectra are consistent with those from observed samples. Distributions formed that include the marginal lines $\left(<5 \sigma_{f i t}\right)$ show that the number of lines continues to increase in accordance with the exponential distribution down to $2.5 \sigma_{f i t}$, and inspection of Figure 6 shows that these marginal lines always correspond to a real feature in the "true" spectrum. We conclude that absorber line counting from this hydrodynamic simulation provides an excellent match to the demographics of the Lyman- $\alpha$ forest at $z=2$.

\subsection{Coincident Lines Between Sight-lines}

The first indications that Lyman- $\alpha$ absorbers were larger than the halos of individual galaxies came from the detection of coincident lines in the spectra of quasar pairs (Dinshaw et al. 1994; Bechtold et al. 1994). Most paired lines of sight experiments define absorbers pairs by choosing a maximum separation in velocity, typically $50-300 \mathrm{~km} \mathrm{~s}^{-1}$, that lines must have in order to be labeled as coincident and therefore physically associated. The operational definition of a matching "window" depends on the line density, and it is chosen to minimize the probability of a chance match. A "coincident" pair is two lines that match in velocity closely enough that the probability of a chance match is small. However, the preselection of a velocity match window can potentially lose information, and it makes an implicit assumption about the kinematic state of the absorbing gas (i.e. the amplitude of any velocity shear on that particular transverse scale). A very large number of paired lines of sight can be extracted from the simulations, with many potential absorber pairs at each of the transverse separations. We have designed a matching algorithm that does not depend on any prior definition of a velocity match. This allows an examination of how the coherence 
of the absorbers changes over transverse separations of $33 h_{50}^{-1} \mathrm{kpc}$ to $1 h_{50}^{-1} \mathrm{Mpc}$.

Coincident lines are defined such that coincidences are symmetric. In other words, if there is a line in sightline $A$, its coincident line is the nearest line in velocity space in sightline $B$. However, coincident lines are also selected starting with sightline $B$ and matching to sightline $A$. An absorber is not identified as a coincident line unless it is a reversible match both from $A$ to $B$ and from $B$ to $A$. This procedure will of course result in lines that are unmatched in both sight-lines. We account for the fact that the simulation cube is periodic by matching across the ends of each simulation spectrum. To discuss line matching statistics, we have chosen to use the single realization of the simulation spectra with $S N R=30$ and $\Gamma_{r e s}=80 \mathrm{~km} \mathrm{~s}^{-1}$. This most closely mimics the observational spectra of the three quasar pairs at $z=2$ that we will analyze in Paper II. Absorber matches were found for 300 primary sight-lines — seven extracted spectra make up each set. The fraction of matched lines is $87 \%, 73 \%, 65 \%, 61 \%, 59 \%$ and $58 \%$ for transverse separations $33,100,233,400,667,1000$ $h_{50}^{-1} \mathrm{kpc}$, respectively. The fraction of matched lines for random lines of sight formed by pairing lines of sight from the x-projection with lines of sight from the z-projection is $57 \%$.

Figure 11 illustrates the line-matching procedure. Figure $11 a$ is merely a test of the algorithm. Figure $11 b$ shows the effect of noise and subsequently slight differences in the degraded spectra on the scatter between the equivalent widths of matched lines. At a $S N R=30$, only $3 \%$ of the lines fail to match due to fluctuations added by random noise. Figure $11 c$ shows that there can be substantial differences in line strength even across a transverse scale of $33 h_{50}^{-1} \mathrm{kpc}$, which is below the resolution of the simulation where intrinsic differences must be small. Nevertheless, the formal correlation is highly significant. Figure $11 d$ shows the null experiment, where absorbers detected in 300 primary sight-lines are matched against 300 randomly selected sight-lines from an orthogonal projection of the simulation. This ensures that any two features at similar wavelengths are separated by 
10-20 $h_{50}^{-1} \mathrm{Mpc}$ in space, and so are expected to be uncorrelated.

In Figure 11b, the scatter is larger than anticipated due to the addition of noise because the line selection procedure must deal with issues of deblending and continuum fitting. The very few outliers (out of 924 line pairs) are mostly caused by the situation where the addition of noise to a marginally resolved feature causes it to be fit with two components in one sightline but only one in the other. At first sight, the large scatter in Figure $11 c$ is more surprising, because the transverse separation is at the limit of resolution of the simulation. The scatter comes about because the mapping from real space to spectral space in complex. Peculiar gas motions affect the optical depth both by shifting line centers and by changing line profiles due to velocity gradients (Bi \& Davidsen 1997). The fact that our line fitting software is responding to real physical differences in opacity can also be seen from the analytic modelling of Viel et al. (2001). They show calculated Lyman- $\alpha$ spectra at $z=2.15$ with noticeable differences on transverse scales as small as $60 h_{50}^{-1} \mathrm{kpc}$, where a significant fraction of lines fit with Voigt profiles have column densities that differ by more than a factor of two. Taken together with the added effects of noise, the scatter in Figure $11 c$ can be readily understood.

A major result of this paper is shown in Figure 12. This shows matched or coincident lines plotted against the velocity difference of the line pair for the six transverse separations with respect to the PLOS. (Of course, it is possible to generate many transverse separations between 0 and $1000 h_{50}^{-1} \mathrm{kpc}$ using these spacings, but the choices in Figure 12 illustrate the coherence phenomenon adequately.) Given the mean line spacing of $850 \mathrm{~km} \mathrm{~s}^{-1}$ (for a $5 \sigma$ line), the line matching algorithm becomes aliased at $425 \mathrm{~km} \mathrm{~s}^{-1}$, so matches with separations above this value are not physically meaningful.

The most sensitive test of absorber coherence uses the fact that truly coincident lines will have a small velocity separation between sight-lines, where random matches (not phys- 
ical associations) will have a larger range of velocity differences. Figure 12 suppresses the information on homogeneity by plotting the average of the equivalent widths of the paired absorbers against the velocity splitting of the pair. The distribution of the velocity splittings of matched pairs changes quite dramatically with transverse separation. To quantify this, we plot the cumulative distribution of the velocity splittings for the paired absorbers in Figure 13. To show the expectation for a random set of absorbers with no physical association between the sight-lines, we also form the cumulative distribution for pairings between spectra extracted from orthogonal projections through the simulation volume. Any two paired absorbers in this case will have a physical separation of $\sim 10-20 h_{50}^{-1} \mathrm{Mpc}$ and so are not anticipated to show coherence (the dashed lines in Figure 13). A K-S test demonstrates that there is an excess of small velocity splittings, and therefore detectable coherence, up to about $500 h_{50}^{-1} \mathrm{kpc}$.

A typical method of examining the homogeneity of Lyman- $\alpha$ absorbers over various transverse separations is to plot the equivalent widths of each line in a matched pair. However, noise and intrinsic differences in the absorbing regions imprint themselves on a spectrum and affect the line fitting algorithm in a complex way, so that apparently similar spectra can yield coincident line pairs with substantially different equivalent widths.

To see if line matching depends on line strength, we formed distributions of equivalent widths for both the paired lines as well as the unpaired lines. A K-S test shows these distributions are drawn from the same distribution. Even though there are many more weak (lower column density) lines than strong (higher column density) lines, the degree of coherence on any particular transverse scale does not depend on line strength. However, the fraction of matched lines decreases with increasing transverse scale; a clear demonstration that coherence is being lost as the scale approaches $1 h_{50}^{-1} \mathrm{Mpc}$. 


\subsection{Comparison with Continuous Statistics}

Up until now we have been examining the coherence of the Lyman- $\alpha$ absorbers using the highest density peaks that are fitted as lines. Clustering or coherence may also be analyzed through the use of continuous statistics that leverage the flux information in every independent point of the spectra. Various types of correlation measures are well described in Cen et al. (1998). We define the two-point correlation function (TPCF) of the normalized absorbed flux, $F(v)$, along neighboring lines of sight as

$$
\xi_{\text {flux }}(v)=\frac{\left\langle F_{1}\left(v+v^{\prime}\right) F_{2}\left(v^{\prime}\right)\right\rangle}{\langle F\rangle}-1,
$$

where $F_{1}$ and $F_{2}$ refer to fluxes along the two lines of sight.

Figure 14 shows the TPCF for each of the transverse separations 33, 100, 233, 400, 667, and $1000 h_{50}^{-1} \mathrm{kpc}$. We compute the auto-correlation function, which is the PLOS correlated with itself $\left(F_{1}=F_{2}\right.$ in Equation 5$)$, and this is shown as the curve with the highest amplitude. We also compute the TPCF for lines of sight that are randomly associated by correlating the PLOS from spectra extracted from the x-projection with PLOS from the z-projection.

The TPCF for discrete absorbers in a line-counting experiment is computed for all transverse separations by comparing the number of observed nearest neighbor pairs, $N_{o b s}$, with the number of pairs formed from random lines of sight, $N_{\text {ran }}$ as follows:

$$
\xi_{\text {pair }}=\frac{N_{\text {obs }}}{N_{\text {ran }}}-1
$$

The random lines of sight are the PLOS from the $\mathrm{x}$ - and z- projections. Figure 14 shows the correlation functions for each of the six transverse separations. The amplitude of the TPCF for each method decreases to approach the curve for random demonstrating that coherence can be measured using line-fitting methods. In Paper II, we will illustrate the different types of information that are gleaned by using continuous flux statistics and line-counting 
techniques, and we will quantify the amount of data required to detect coherence using line counting.

\section{SUMMARY}

This paper has established techniques for the intercomparison of quasar spectra and spectra extracted from cosmological simulations, recognizing that observations are traditionally interpreted in terms of line-counting, while simulations offer a direct measure of neutral hydrogen opacity at every resolution element. This work anticipates a series of direct comparisons using space- and ground-based observations of quasar pairs and groups. Initial tests have been carried out on the Lyman- $\alpha$ forest at $z=2$, with simulation spectra degraded to span the range of observational quality of typical ground and space-based data. The main results are as follows:

(1) Simulators measure opacity with respect to a pre-defined continuum, while observers must determine a quasar continuum that is not known a priori, in the presence of noise and emission features. Software has been designed that robustly measures continua both for the small wavelength (redshift) range of simulations and for the long wavelength range of typical observations. The systematic underestimate of opacity ranges from $\Delta \tau=0.02$ for $S N R=100, \Gamma_{\text {res }}=5 \mathrm{~km} \mathrm{~s}^{-1}$ to $\Delta \tau=0.06$ for $S N R=10, \Gamma_{\text {res }}=300 \mathrm{~km} \mathrm{~s}^{-1}$.

(2) A fully automated procedure has been developed for the detection and selection of absorption features that produces reliable results for observational data having $S N R=10$ to 30 and $\Gamma_{\text {res }}=80$ to $300 \mathrm{~km} \mathrm{~s}^{-1}$. The techniques are robust even in extended regions of strong absorption. A direct comparison with published quasar surveys shows that the number density and equivalent width distribution of absorption lines at $z=2$ agrees with measurements of spectra extracted from an SPH simulation. 
(3) A technique has been established to match absorption lines between adjacent lines of sight. The match rate of coincident lines is used to search for coherence in the absorbing gas on transverse scales up to $1 h_{50}^{-1} \mathrm{Mpc}$. The two-point correlation function of matched pairs reveals a significant excess out to transverse scales of $\sim 500 h_{50}^{-1} \mathrm{kpc}$, indicating the detection of Lyman- $\alpha$ coherence on this scale. Most of the signal of excess pairs occurs with velocity splittings of $<100 \mathrm{~km} \mathrm{~s}^{-1}$, indicating that the velocity field is quiet. The coherence signal measured with line counting and matching techniques agrees well with results from a two-point flux correlation analysis, which uses all the information in the simulation spectra.

We are particularly grateful to Tom Aldcroft, who made available the code that formed the core of the software suite described in this paper, and Jennifer Scott, who shared her thesis data. We acknowledge useful discussions with Romeel Davé, Craig Foltz, Jane Charlton, and Rupert Croft. This work was supported by NASA Astrophysical Theory Grants NAG5-3922, NAG5-3820, and NAG5-3111, by NASA Long-Term Space Astrophysics Grant NAG5-3525, and by the NSF under grants ASC93-18185, AST-9803072, and AST-9802568. 


\section{REFERENCES}

Aldcroft, T. 1993, Ph.D. Thesis, Stanford University

Aguirre, A. et al. 2001, ApJ, 556, 11

Aguirre, A. et al. 2001, ApJ, submitted [astro-ph/0006345]

Bahcall, J.N. et al. 1993, ApJS, 87, 1

Bechtold, J., Crotts, A.P.S., Duncan, R.C., \& Fang, Y. 1994, ApJ, 437, L83

Bi, H. G. 1993, ApJ, 405, 479

Bi, H. G. \& Davidsen, A. F. 1997, ApJ, 479, 523

Cen, R., Miralda-Escudé, J., Ostriker, J.P., \& Rauch, M. 1994, ApJ, 437, 9

Cen, R., Phelps, S., Miralda-Escudé, J., \& Ostriker, J.P. 1998, ApJ, 496, 577

Cen, R., \& Ostriker, J.P. 1999, ApJ, 519, L109

Croft, R.A.C., Weinberg, D.H., Katz, N., \& Hernquist, L. 1998, ApJ, 495, 44

Croft, R.A.C., Weinberg, D.H., Pettini, M., Hernquist, L., \& Katz, N. 1999, ApJ, 520, 1

Croft, R.A.C., et al. 2001, submitted [astro-ph/0012324]

Crotts, A.P.S., \& Fang, Y. 1998, ApJ, 502, 16

Davé, R., Hernquist, L., Weinberg, D.H., \& Katz, N. 1997, ApJ, 477, 2

Davé, R., Hellsten, U., Hernquist, L., Katz, N., \& Weinberg, D.H. 1998, ApJ, 509, 661

Davé, R., Hernquist, L., Katz, N. \& Weinberg, D.H. 1999, ApJ, 511, 521 D.H., \& Katz, N. 1997, ApJ, 477, 2 
Dinshaw, N. Impey, C.D., Foltz, C.B., Weymann, R.J., \& Chaffee, F.C. 1994, ApJ, 437, L87

Dinshaw, N., Foltz, C.B., Impey, C.D., \& Weymann, R.J. 1998, ApJ, 494, 567

Gnedin, N.Y., \& Hui, L. 1996, ApJ, 472, L73

Gnedin, N.Y. 1998, MNRAS, 299, 392

Gunn, J.E., \& Peterson, B.A. 1965, ApJ, 142, 1633

Haehnelt, M, \& Steinmetz, M. 1998, MNRAS, 298, L21

Hellsten, U. et al. 1997, ApJ, 487, 482

Hernquist, L. \& Katz, N. 1989, ApJS, 70, 419

Hernquist, L., Katz, N., Weinberg, D.H., \& Miralda-Escudé, J. 1996, ApJ, 457, 51

Hu, E.M., Kim, T.-S., Cowie, L.L. Songaila, A., \& Rauch, M. 1995, AJ, 110, 1526

Jannuzi et al. 1998, ApJS, 118, 1

Katz, N., Weinberg, D.H., \& Hernquist, L. 1996, ApJS, 105, 19

Katz, N., Weinberg, D.H., Hernquist, L., \& Miralda-Escudé, J. 1996, ApJ, 457, 57

Kirkman, D., \& Tytler, D. 1997, ApJ, 484, 672

McDonald, P. et al. 2000, ApJ, 543, 1

McGill, C. 1990, MNRAS, 242, 544

Miralda-Escudé, J., Cen, R., Ostriker, J.P., \& Rauch, M. 1996, ApJ, 471, 582

Nusser, A., \& Haehnelt, M. 2000, MNRAS, 313, 587

Penton, S.V., Stocke, J.T., \& Shull, M.J. 2000, ApJS, 130, 121 
Petry, C.E., Impey, C.D., \& Foltz, C.B. 1998, ApJ, 494, 6

Penton, S.V., Shull, M.J., \& Stocke, J.T. 2000, ApJ, 544, 150

Rauch, M., Carswell, R.F., Chaffee, F.H., Foltz, C.B., Webb, J.K., Bechtold, J., \& Green, R.F. 1992, ApJ, 390, 387

Rauch, M., et al. 1997, ApJ, 489, 7

Rauch, M. et al. 1997, ApJ, 481, 601

Rauch, M. 1998, ARA\&A, 36, 267

Ricotti, M., Gnedin, N.Y., \& Shull, J.M. 2000, ApJ, 534, 41

Schaye, J. et al. 2000, MNRAS, 318, 817

Schneider, D.P., et al. 1993, ApJS, 87, 45

Scott, J., Bechtold, J., \& Dobrzycki, A. 2000, ApJS, 130, 37

Tripp, T.M., Savage, B.D., \& Jenkins, E.B. 2000, ApJ, 534, 1

Viel, M., Matarrese, S., Mo, H. J., Haehnelt, M. G., \& Theuns, T. 2001, astro-ph/0105233

Weinberg, D.H., Miralda-Escudé, J., Hernquist, L., \& Katz, N. 1997, ApJ, 490, 564

Weinberg, D. H., Katz, N., \& Hernquist, L. 1998, in ASP Conference Series 148, Origins, eds. C. E. Woodward, J. M. Shull, \& H. Thronson, (ASP: San Francisco), 21

Weinberg, D.H., Croft, R.A.C., Hernquist, L., Katz, N., \& Pettini, M. 1999, ApJ, 522, 563

Weymann, R.J., Rauch, M., Williams, R., Morris, S., \& Heap, S. 1995, ApJ, 438, 650

Weymann, R.J, et al. 1998, ApJ, 506, 1 
York, D.G. et al. 2000, AJ, 120, 1579

This preprint was prepared with the AAS LATEX macros v5.0. 
Table 1. Comparison of Number Density of Lyman- $\alpha$ Absorbers

\begin{tabular}{crrrrrr}
\hline \hline Sample & $S N R$ & $\Gamma_{\text {res }}{ }^{\mathrm{a}}$ & $W_{\text {lim }}{ }^{\mathrm{b}}$ & $\left(\frac{d N}{d z}\right)_{\text {sim }}$ & $\left(\frac{d N}{d z}\right)_{o b s}{ }^{\mathrm{c}}$ & $\left(\frac{d N}{d z}\right)_{o b s}{ }^{\mathrm{d}}$ \\
$(1)$ & $(2)$ & $(3)$ & $(4)$ & $(5)$ & $(6)$ & $(7)$ \\
& & & & & & \\
\hline & & & & & & \\
1 & 30 & 80 & 0.05 & $170 \pm 3$ & $143 \pm 11$ & $128_{-63}^{+125}$ \\
2 & 30 & 300 & 0.05 & $75 \pm 4$ & $\ldots$ & $\ldots$ \\
3 & 10 & 80 & 0.17 & $92 \pm 4$ & $108 \pm 2$ & $81_{-40}^{+79}$ \\
4 & 10 & 300 & 0.17 & $38 \pm 3$ & $\ldots$ & $\ldots$ \\
\hline
\end{tabular}

${ }^{a}$ Resolution of the degraded simulation spectra as described by the FWHM of a Gaussian distribution in $\mathrm{km} \mathrm{s}^{-1}$.

${ }^{\mathrm{b}}$ The limiting rest equivalent width for an isolated $5 \sigma_{\text {det }}$ absorber.

${ }^{\mathrm{c}}$ The number of absorbers per unit redshift obtained by counting the number of lines in the sample of Scott, Bechtold, \& Dobrzycki (2000) in the redshift range $2<z<2.1$ with $5 \sigma_{f i t}$. The errors are $1 \sigma$ Poisson errors; systematic uncertainties are likely to be larger.

${ }^{d}$ The number of absorbers per unit redshift computed from the absorber evolution model of Scott, Bechtold, \& Dobrzycki (2000). The errors correspond to the $1 \sigma$ errors on $\gamma$, where the integrated evolution function is specified by Equation 4 in the text. 


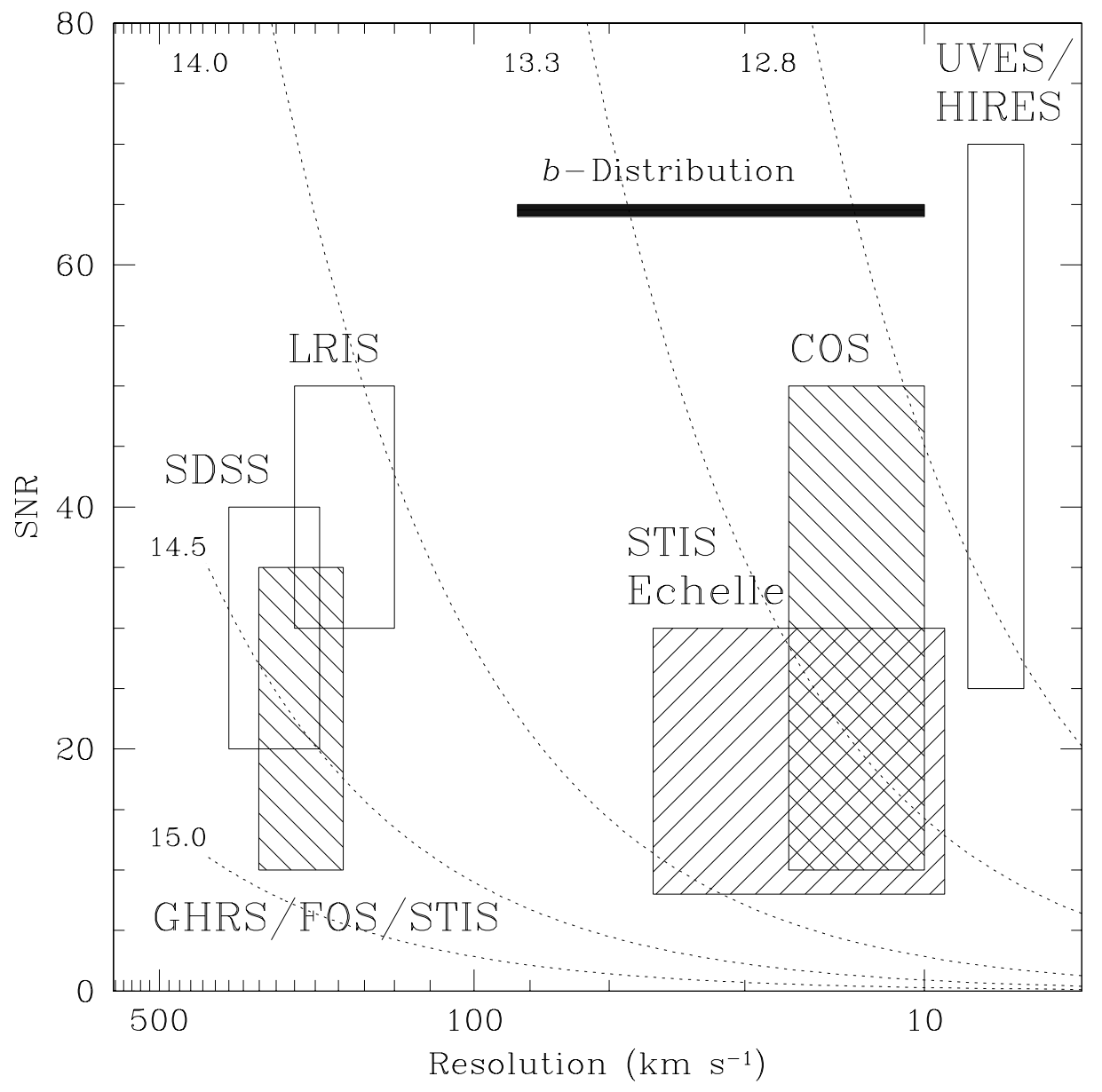

Fig. 1. - A schematic view of the range in $S N R$ and resolution of the major examples of quasar spectra from pioneering observational facilities. Open boxes refer to ground-based observations of Lyman- $\alpha$ with $z \gtrsim 1.6$; shaded boxes refer to the unique capabilities of HST at lower redshifts. The horizontal bar shows the (redshift-independent) range of Doppler parameters, as measured with echelle data. The dotted lines show the detection limits for a $5 \sigma_{\text {det }}$ line having hydrogen column density as labeled, $\log N=15.0,14.5,14.0,13.3,12.8$ atoms $\mathrm{cm}^{-2}$. The detection limits are computed assuming the spectral dispersion is one-third of the instrumental resolution. 

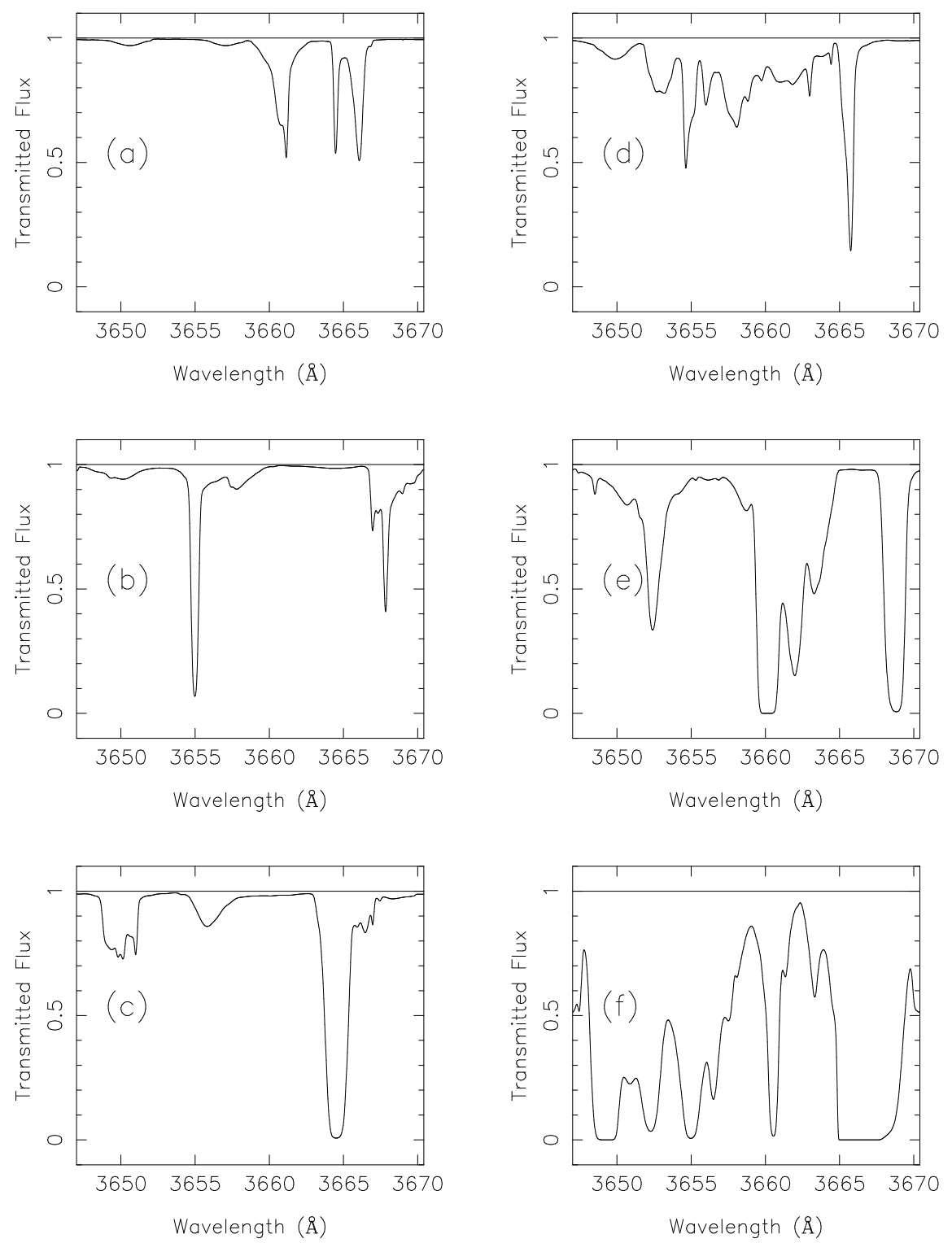

Fig. 2.- Examples of undegraded (i.e. raw) spectra extracted from the simulation. The mean transmitted flux at $z=2$ is 0.83 ; panels $(c)$ and $(d)$ have a typical amount of absorption. The mean opacities from panels $(a)$ to $(f)$ are $0.06,0.07,0.13,0.15,0.34,1.06$. The percent of simulated spectra with opacity less than that shown in panels $(a)$ through $(f)$ is $1 \%, 6 \%$, $37 \%, 47 \%, 95 \%$, and $99 \%$, respectively. 

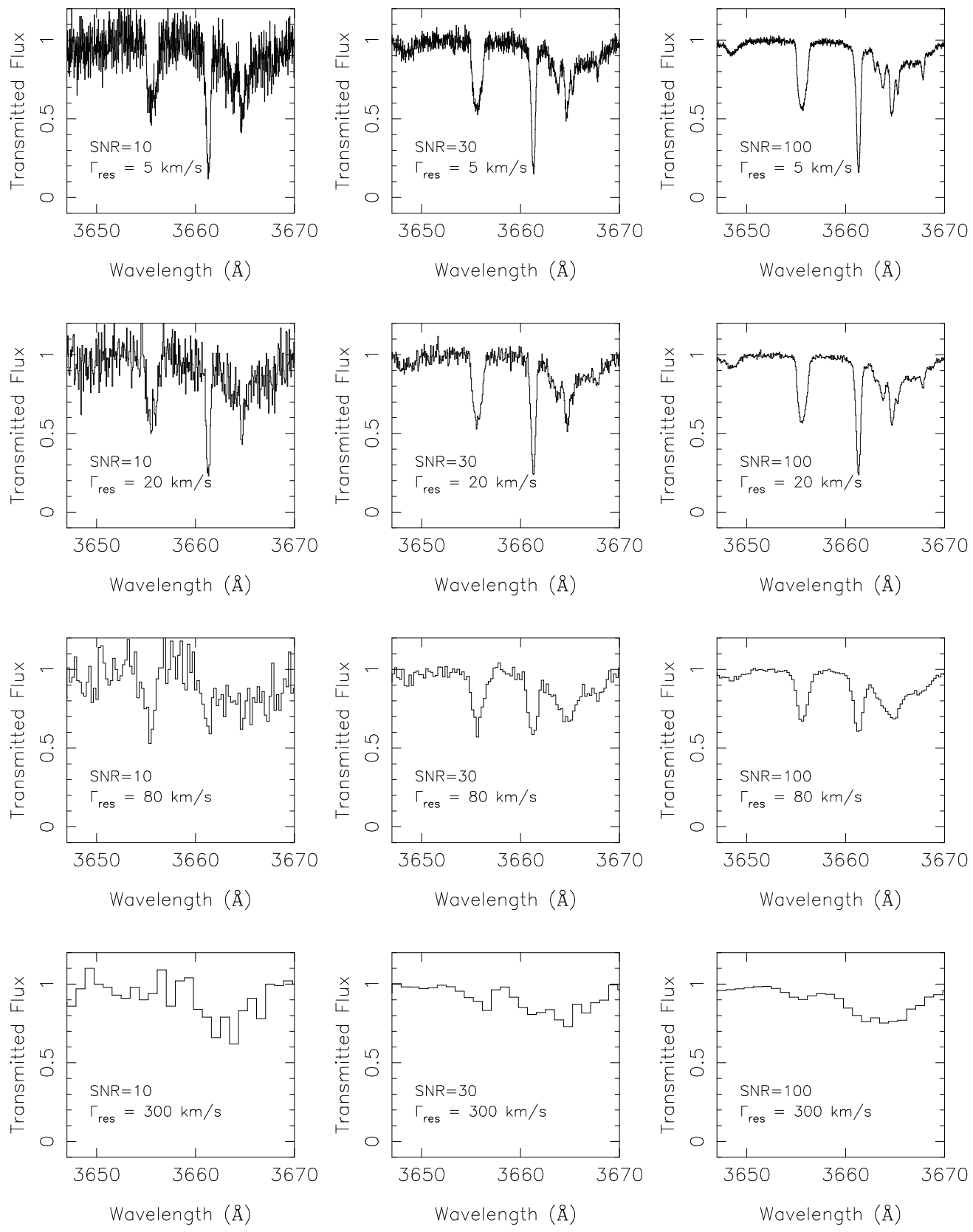

Fig. 3. - Two spectra extracted from the simulation, each of typical opacity at $z=2$. The twelve panels in each case show the spectrum degraded to $S N R=10,30$, and 100, and $\Gamma_{r e s}=5,20,80$, and $300 \mathrm{~km} \mathrm{~s}^{-1}$. The top right panel is a reasonable facsimile of the original spectrum. 

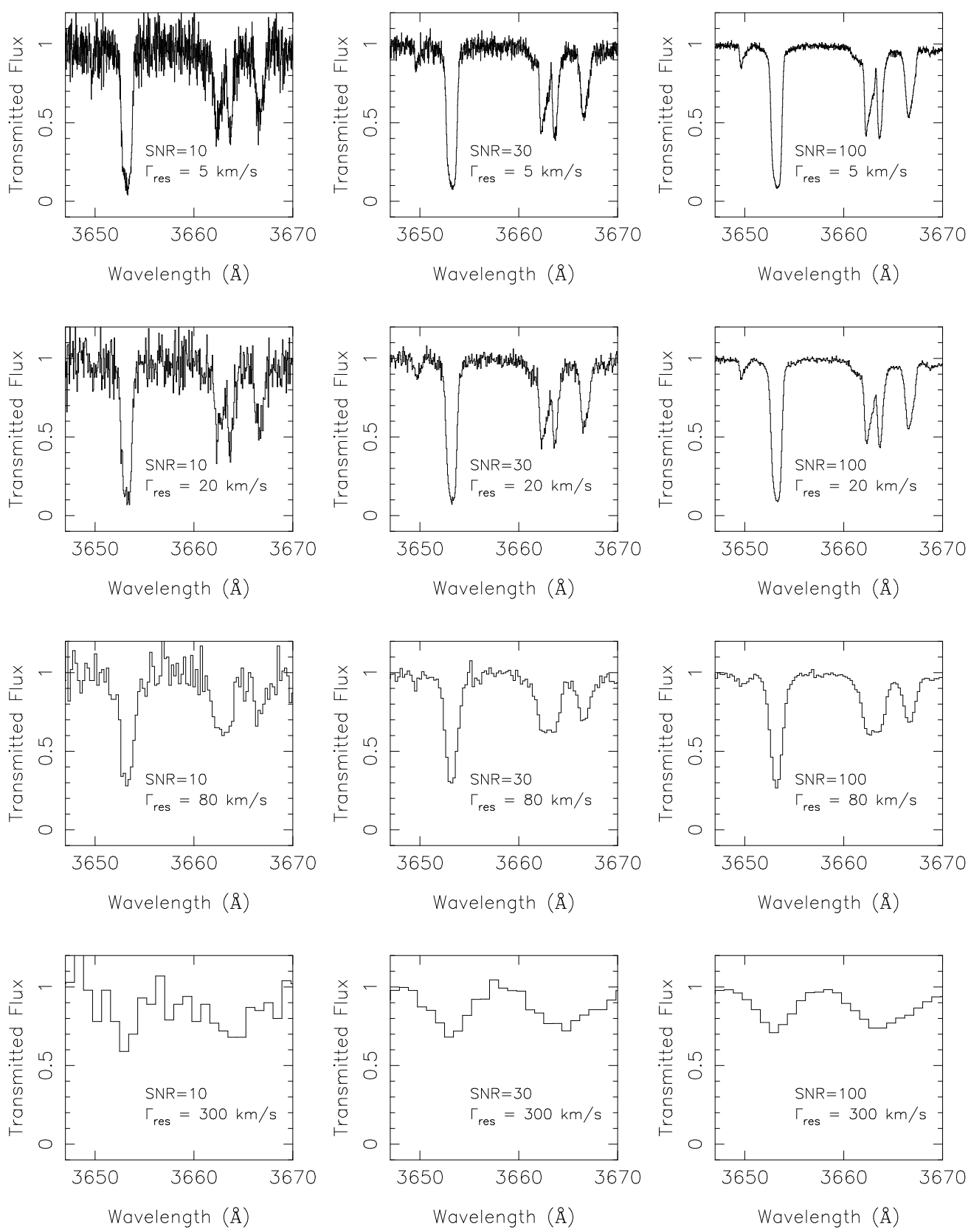

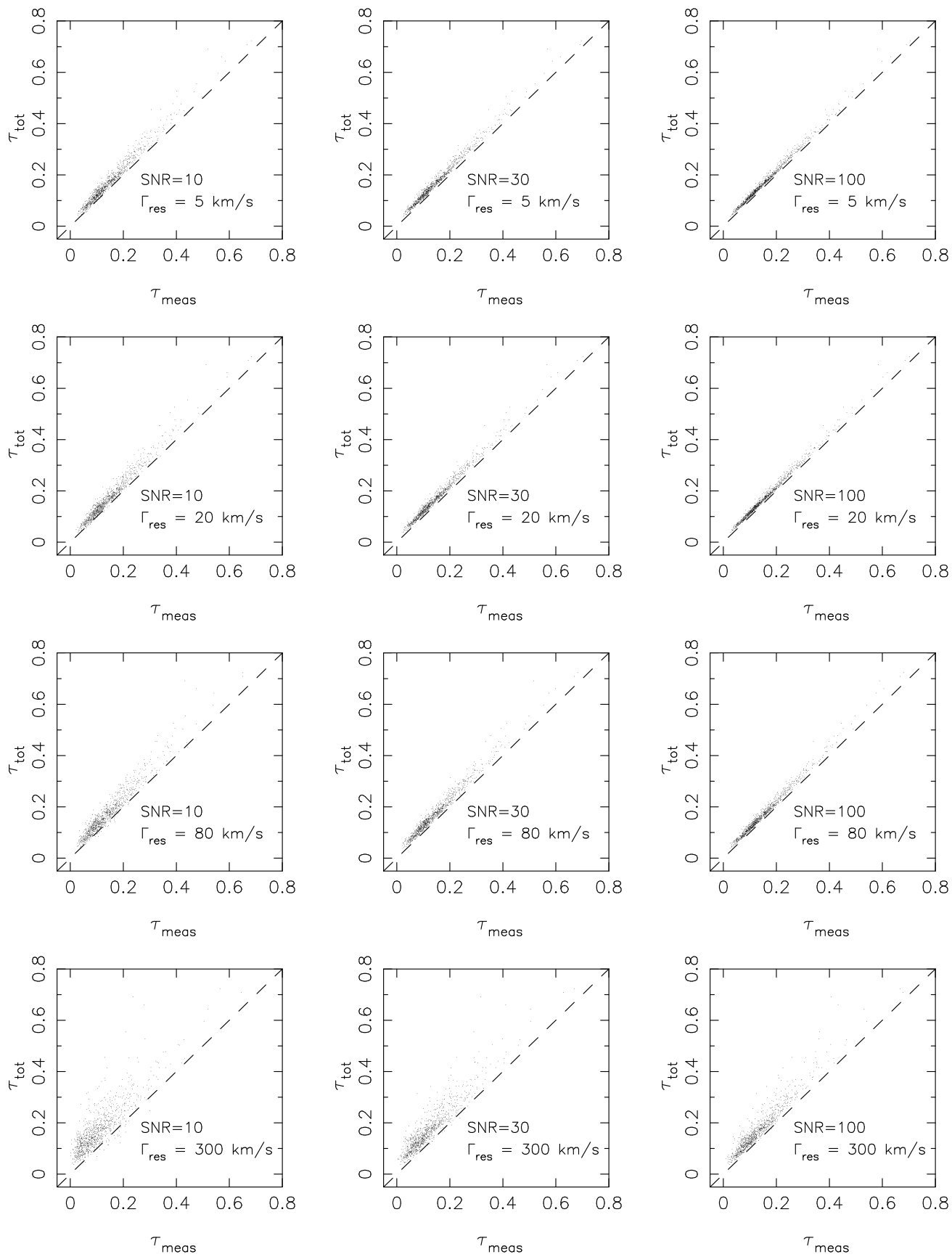

Fig. 4.- An illustration of how the underestimate of opacity due to continuum fitting depends on both $S N R$ and resolution. The mean opacity from each undegraded spectrum is plotted against the mean opacity measured relative to the fitted continuum for each degraded spectrum. Each panel represents a different combination of $S N R$ and resolution; the layout is the same as Figure 3. 



Fig. 5.- The opacity decrement from the continuum fitting procedure applied to 1000 spectra extracted from the simulation. The quantity $\Delta \tau$ is the difference between the continuum level of an input spectrum (raw, undegraded) and the fitted continuum to a degraded spectrum, converted into opacity. 


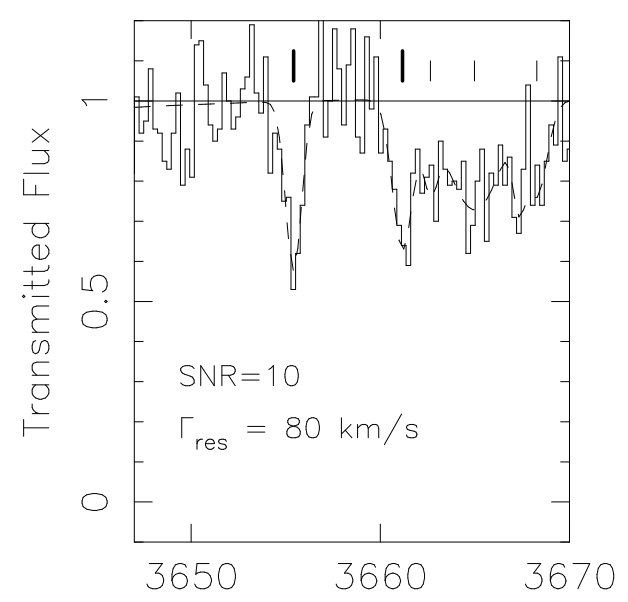

Wavelength $(\AA)$

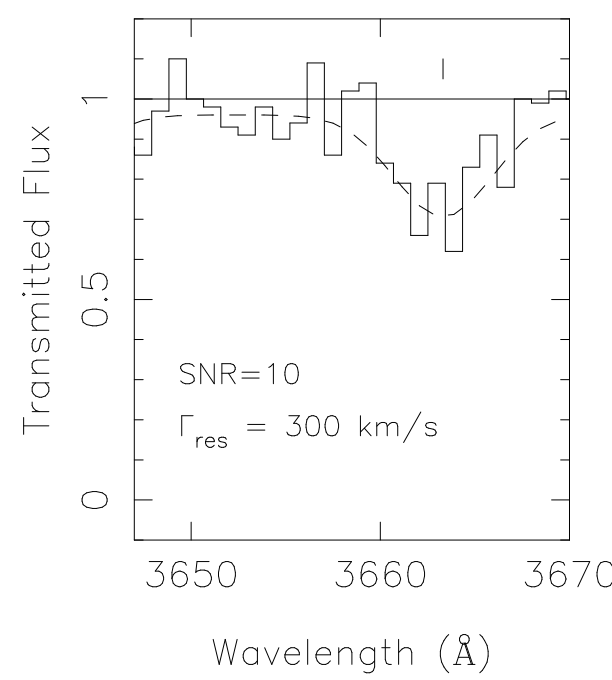



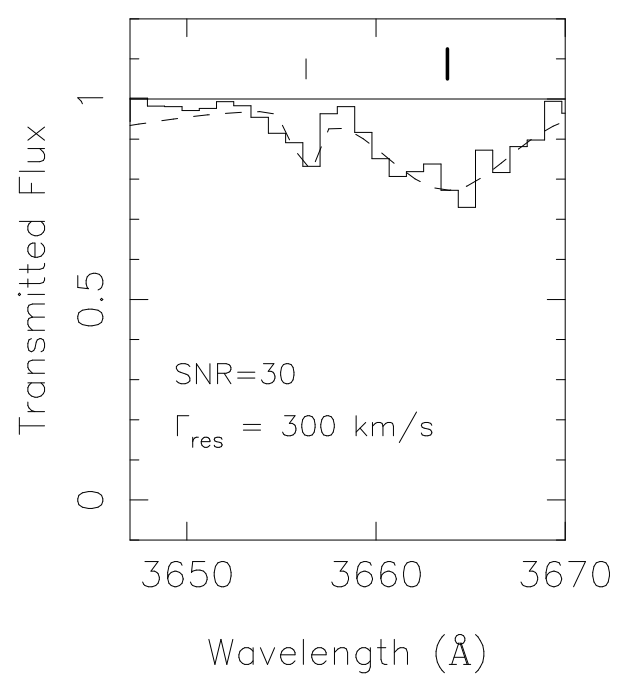

Fig. 6.- An illustration of the line-fitting procedure for the two extracted spectra from Figure 3, in each of the realizations $S N R=10$ and $30, \Gamma_{r e s}=80$ and $300 \mathrm{~km} \mathrm{~s}^{-1}$. Fitted continuum and superimposed lines are shown by a dashed line, and line centers of absorbers with strength of at least $5 \sigma_{\text {det }}$ are shown by tick marks. Longer thicker ticks denote lines with secure reliability $\geq 5 \sigma_{f i t}$ and shorter thinner ticks mark lines with marginal reliability of $<\sigma_{f i t}$. 

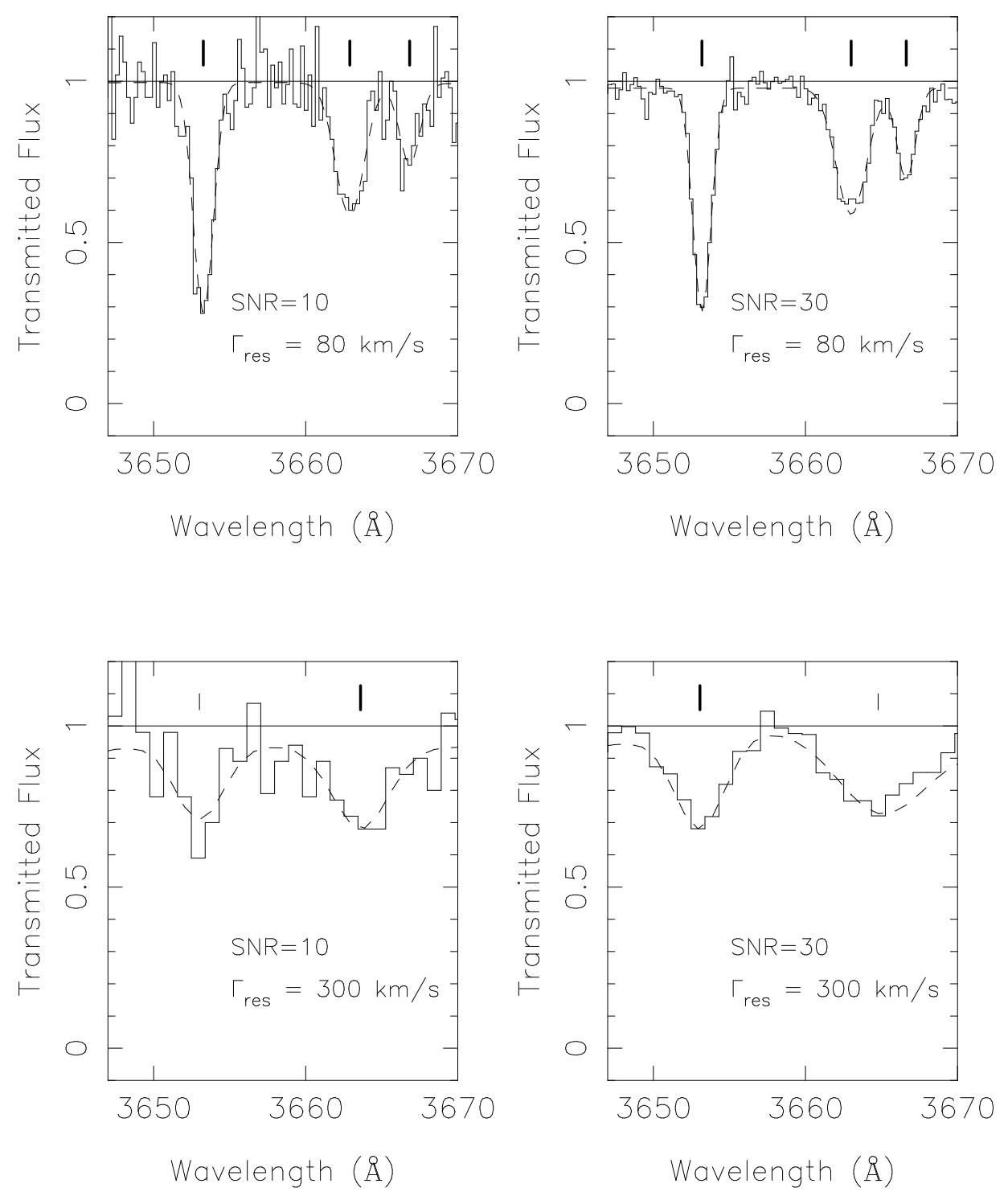

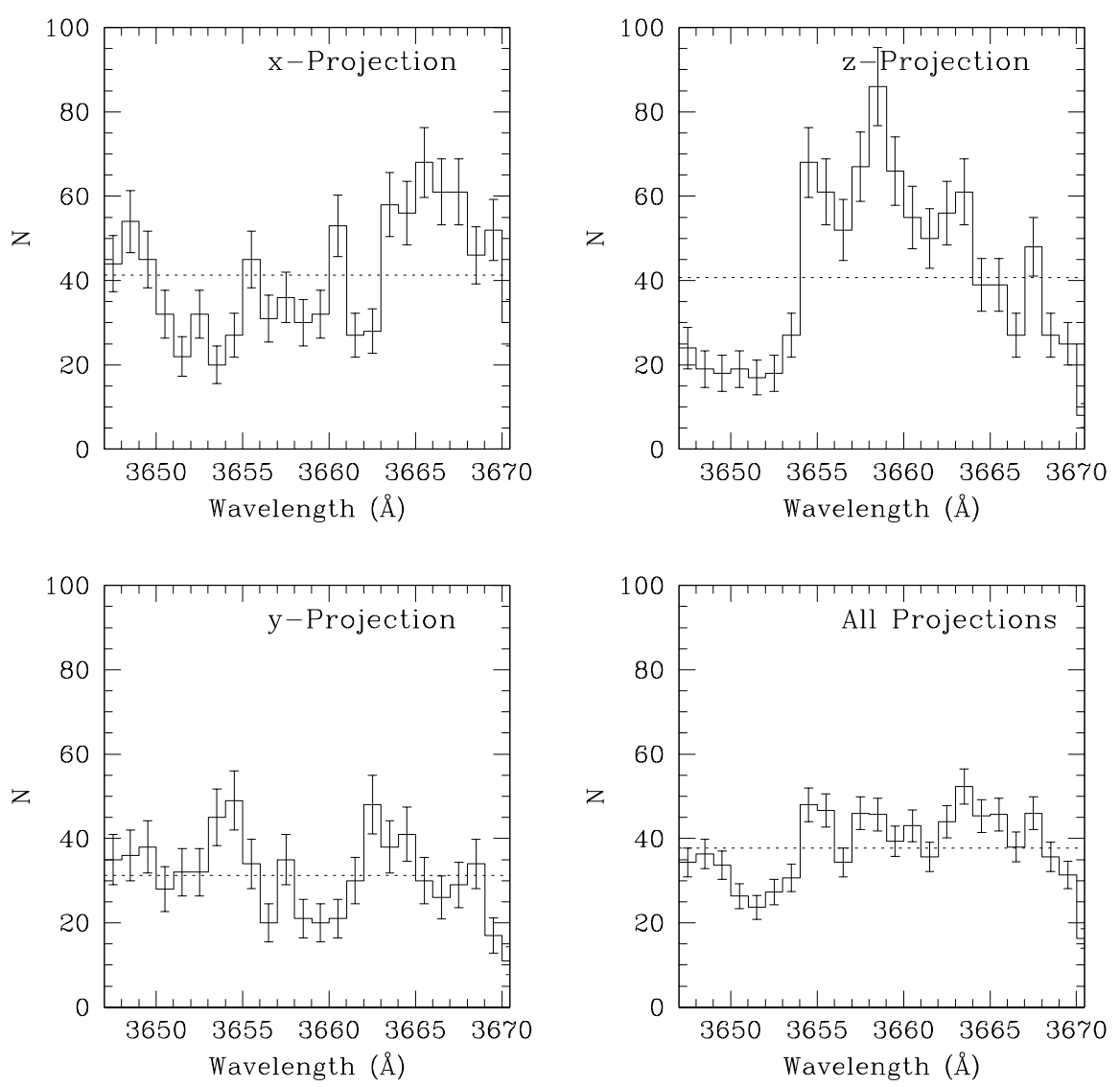

Fig. 7.- The distribution of line centers for absorbers found in 300 PLOS for the $S N R=30$, $\Gamma_{r e s}=80 \mathrm{~km} \mathrm{~s}^{-1}$ realization for the $\mathrm{x}-, \mathrm{y}^{-}$, and $\mathrm{z}^{-}$projections. The dotted line shows the mean of the distribution. The individual projections reveal coherent, large-scale structures. The average of the individual projections (lower right) shows that the distribution converges toward a uniform distribution as more lines of sight from all projections are combined. 

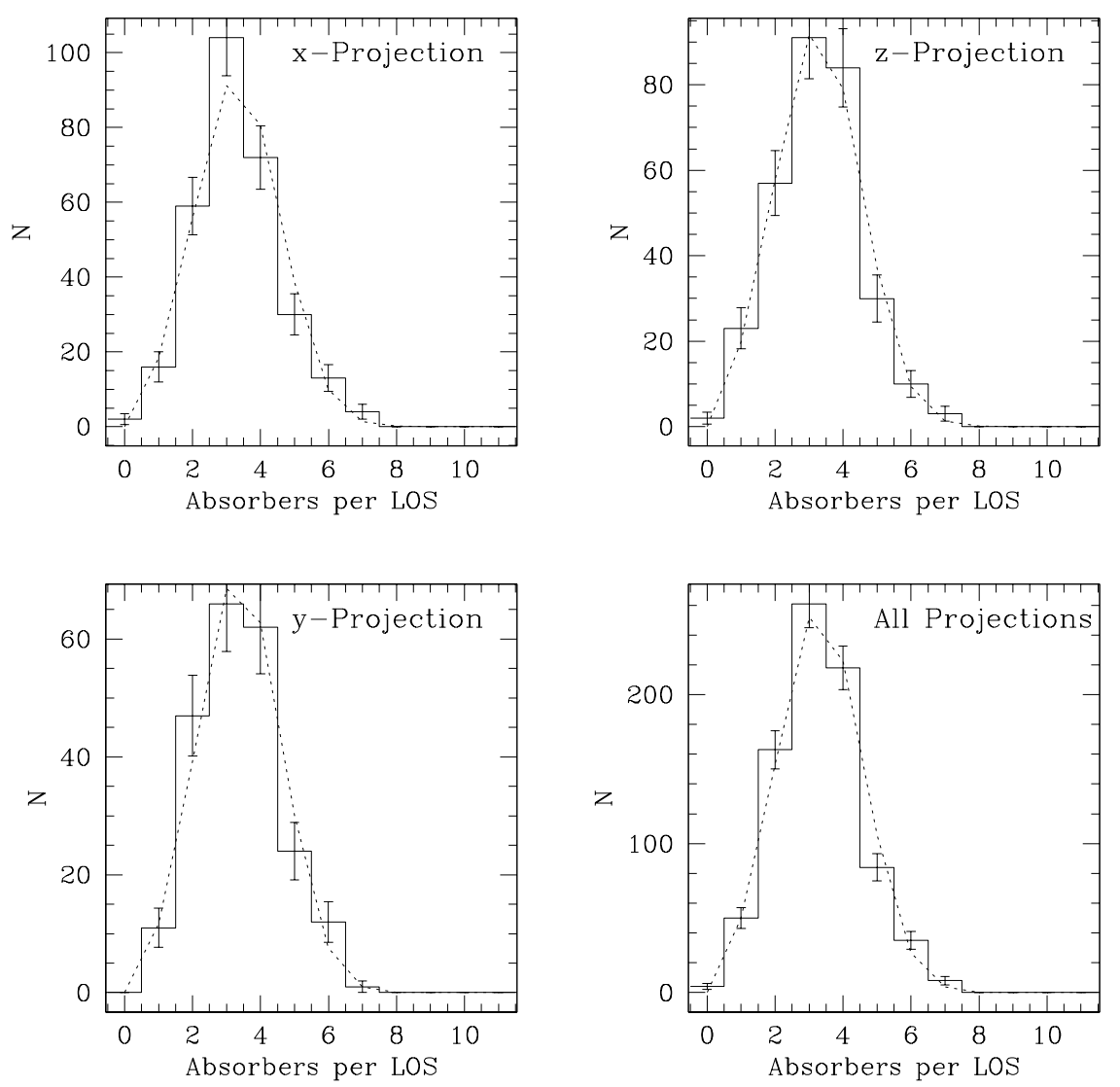

Fig. 8. - The distribution of the number of absorbers per line of sight for 300 PLOS for the $S N R=30, \Gamma_{r e s}=80 \mathrm{~km} \mathrm{~s}^{-1}$ realization for the $\mathrm{x}-, \mathrm{y}-$, and $\mathrm{z}-$ projections. The dotted line is a Gaussian distribution with the mean and standard deviation of the data. The good match to the Gaussian demonstrates that the number of absorbers per line of sight is consistent with a random distribution of absorbers on scales similar to the size of the simulation box. 


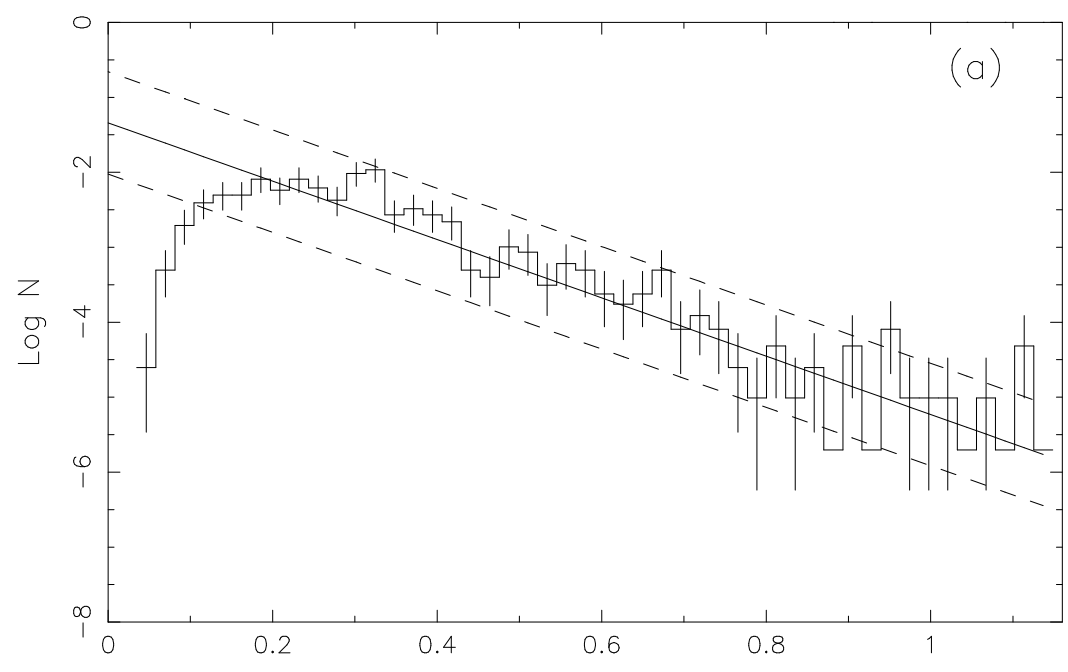

Rest Equivalent Width ( $\AA$ )



Fig. 9.- (a) A histogram of the number of $5 \sigma_{\text {fit }}$ absorbers as a function of rest equivalent width, measured by line-fitting applied to 300 spectra extracted from the simulation degraded to $S N R=10, \Gamma_{\text {res }}=80 \mathrm{~km} \mathrm{~s}^{-1}$. The solid line is the best-fit evolution function to the observed data at $z=2$ from Scott, Bechtold, \& Dobrzycki (2000), with dashed lines showing $1 \sigma$ error bars due to the parameter $\gamma$. (b) Expanded view of the distribution of weak line strengths, with detection significance superimposed. 


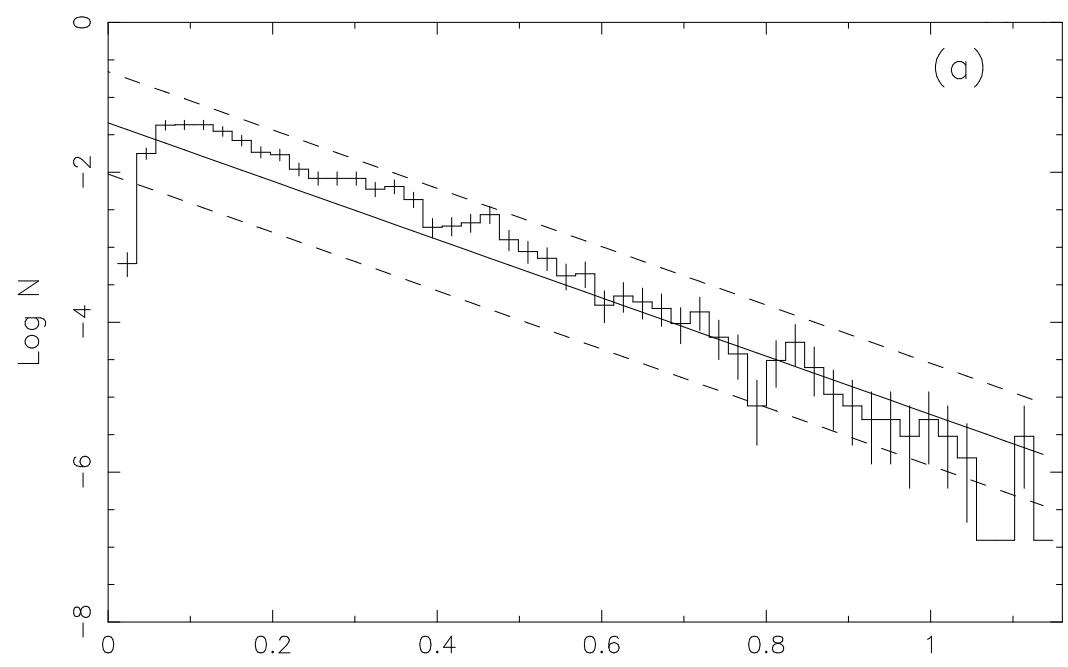

Rest Equivalent Width ( $\AA$ )

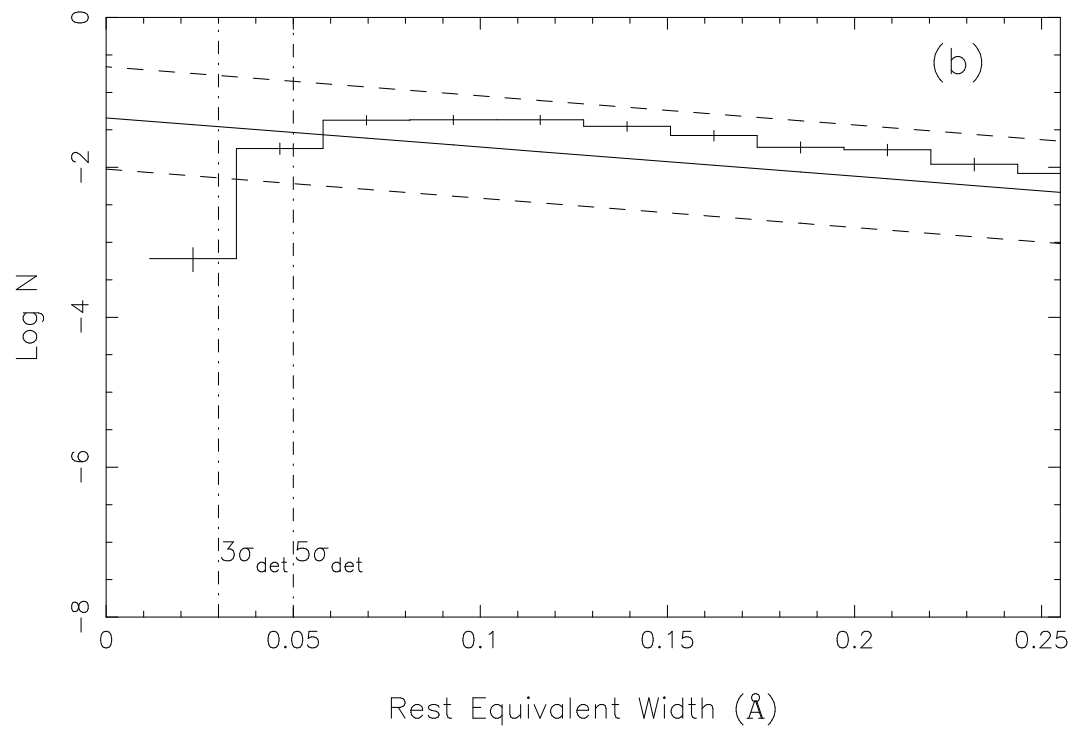

Fig. 10.- (a) A histogram of the number of $5 \sigma_{f i t}$ absorbers as a function of rest equivalent width, measured by line-fitting applied to 1000 spectra extracted from the simulation degraded to $S N R=30, \Gamma_{r e s}=80 \mathrm{~km} \mathrm{~s}^{-1}$. As in Figure 9, the solid line is the best-fit evolution function to observed data at $z=2$ from Scott, Bechtold, \& Dobrzycki (2000), with dashed lines showing $1 \sigma$ error bars due to the parameter $\gamma$. (b) Expanded view of the distribution of weak line strengths, with detection significance superimposed. 

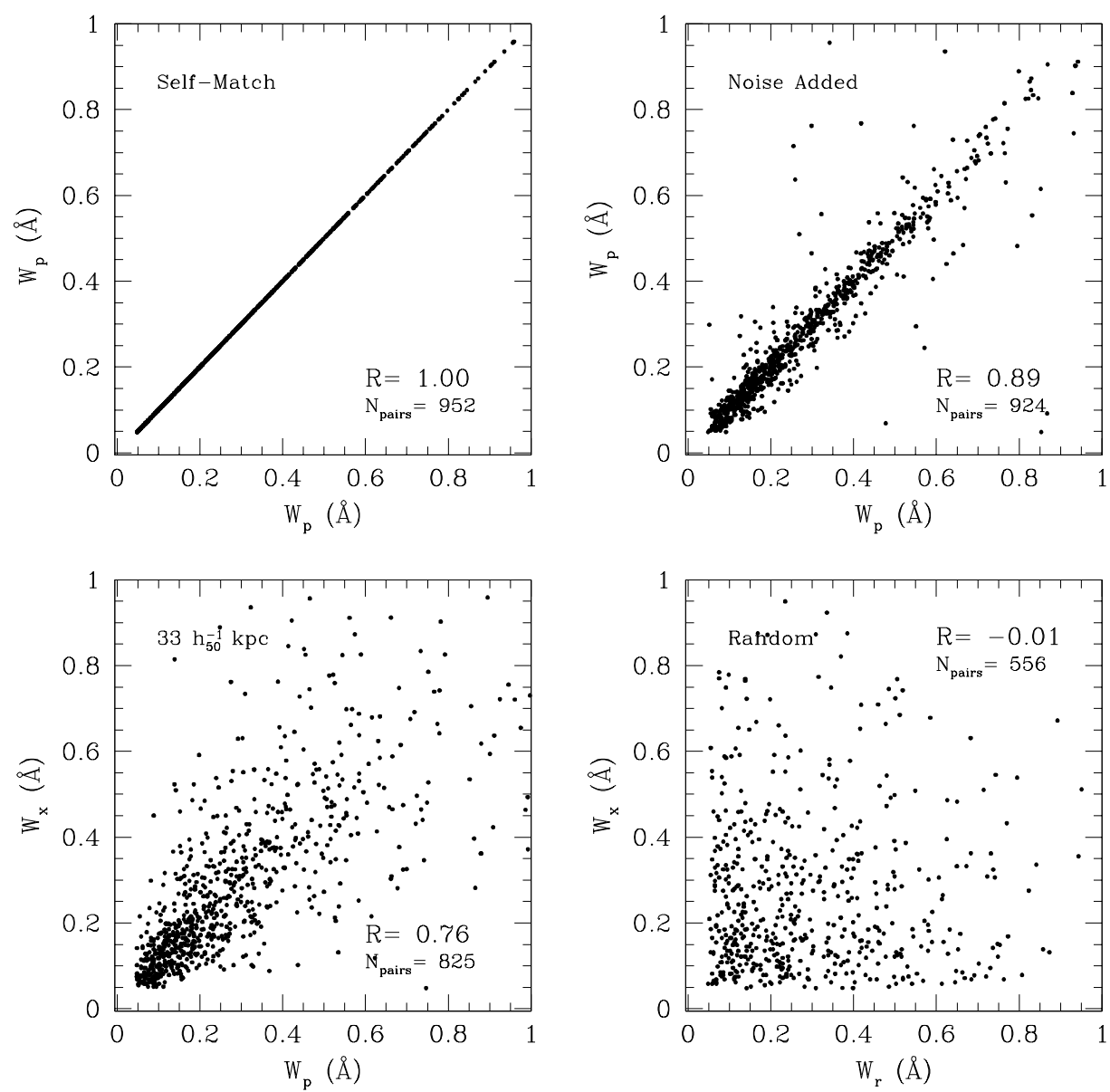

Fig. 11. - A plot of the rest equivalent widths of paired absorbers for the sample where $S N R=30$ and $\Gamma_{\text {res }}=80 \mathrm{~km} \mathrm{~s}^{-1}$. (a) 300 primary lines of sight are matched to themselves as a test of the algorithm, so the correlation is perfect. (b) 300 primary lines of sight are matched to themselves, but with a different noise seed. (c) Matched pairs found for the closest transverse separation of $33 h_{50}^{-1} \mathrm{kpc}$, which approaches the resolution of the simulation, so intrinsic differences in the spectra should be small. (d) 300 primary lines of sight are matched to 300 randomly-selected lines of sight from an orthogonal projection of the simulation. No pairs due to physical coherence are expected in this situation. 

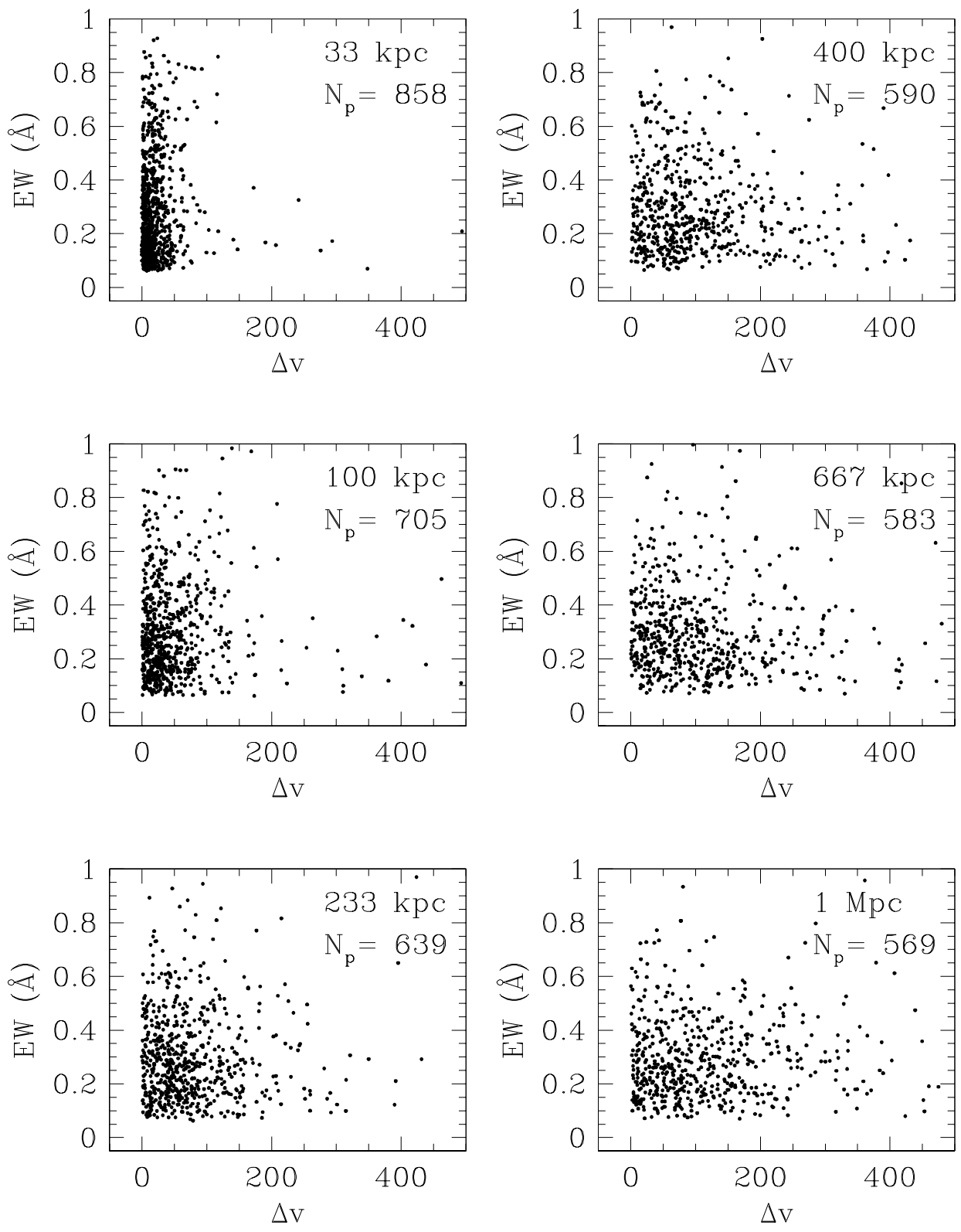

Fig. 12.- The average rest equivalent width for matched absorber pairs plotted against the velocity splitting of the pair for 300 sets of lines of sight at 6 transverse separations. The fraction of matched lines goes down from $87 \%$ for a separation of $33 h_{50}^{-1} \mathrm{kpc}$ to $58 \%$ for a separation of $1000 h_{50}^{-1} \mathrm{kpc}$. The fraction of matched lines for random lines of sight is $57 \%$. 

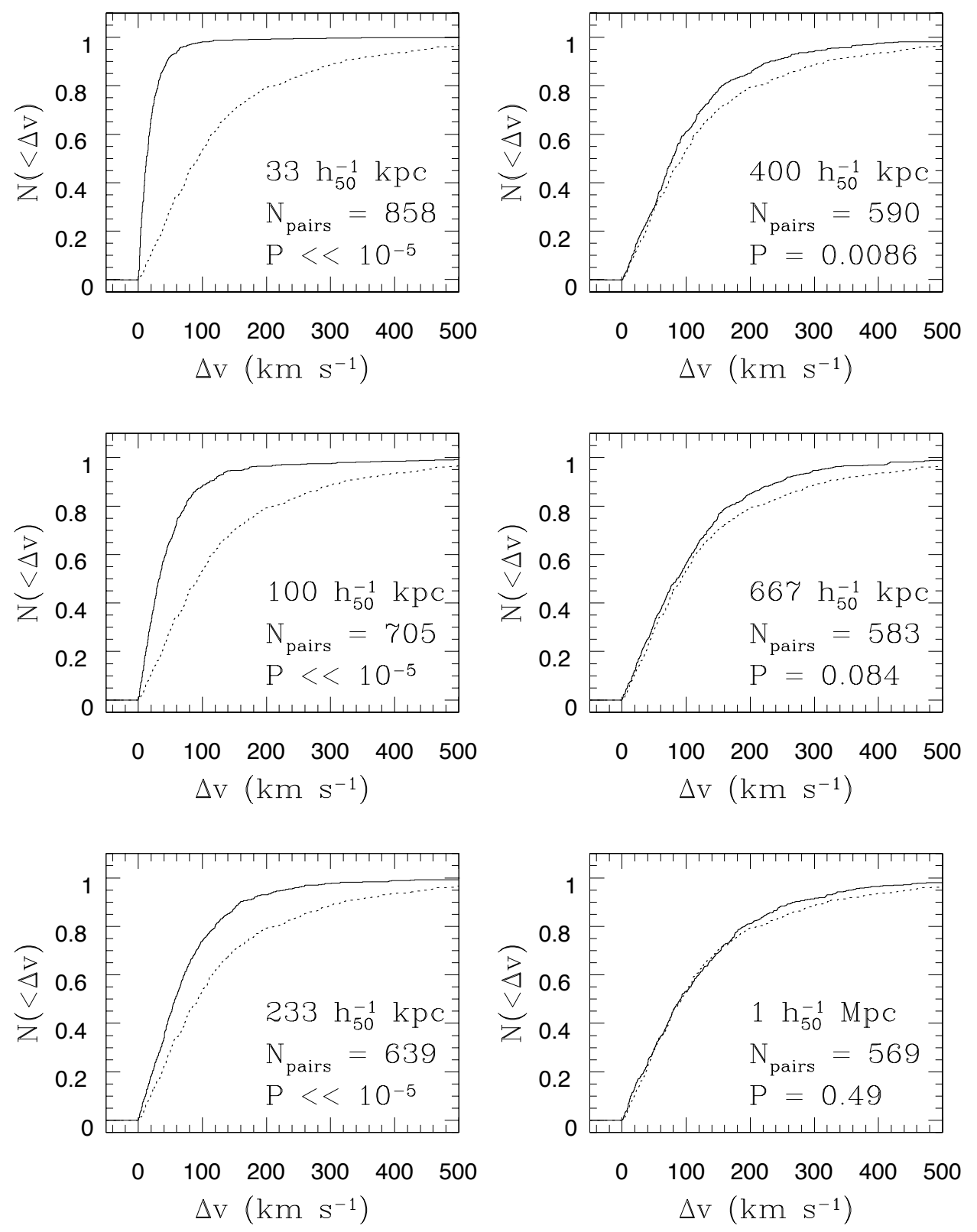

Fig. 13.- The cumulative distribution of velocity splittings for absorber pairs at each transverse separation in the sample (300 sets) is shown by the solid line. The dashed line is the cumulative distribution of absorber pairs formed by pairing x-projection primary lines of sight with z-projection primary lines of sight, approximating the distribution expected for random absorber pairs. 

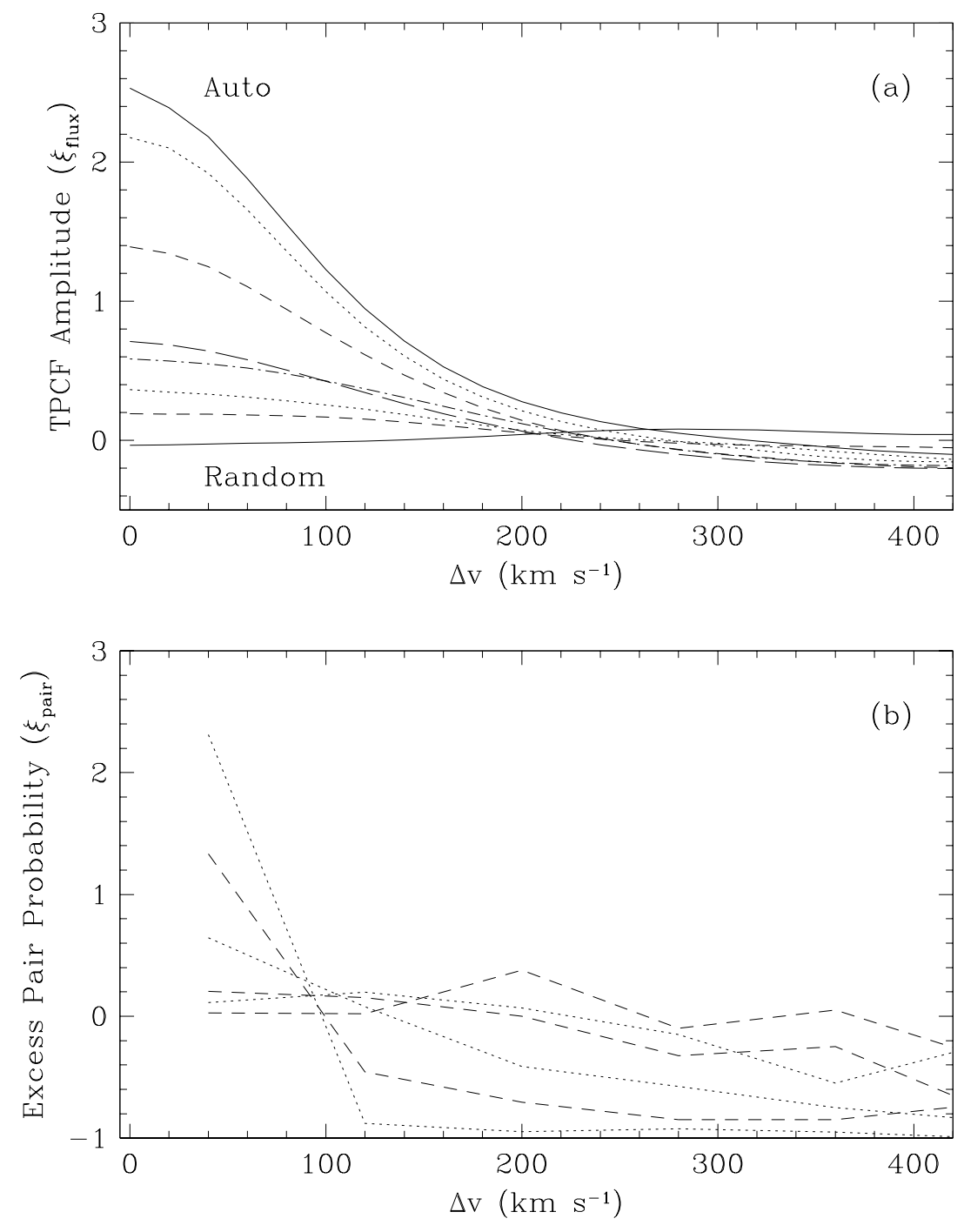

Fig. 14.- (a) The two-point correlation function (TPCF) of the transmitted flux for spectra having transverse separations of $33,100,233,400,667$ and $1000 h_{50}^{-1} \mathrm{kpc}$, which is the sequence from the highest to the lowest curve at $\Delta v=0$. The curve with the highest amplitude is the auto-correlation formed from correlating the PLOS with themselves. The TPCF for random lines of sight is formed by correlating the PLOS of the x-projection with the PLOS of the z-projection. (b) The TPCF for discrete absorber pairs. The curve with the highest amplitude is for a transverse separation of $33 h_{50}^{-1} \mathrm{kpc}$. Curves with subsequently lower amplitudes are for separations of 100, 233, 400, 667 and $1000 h_{50}^{-1} \mathrm{kpc}$. 